\title{
Long-Lived Charge Transfer Excited States in HBC- Polypyridyl Complex Hybrids
}

Anastasia B. S. Elliott ${ }^{1}$, Raphael Horvath², Xue-Zhong Sun², Michael G. Gardiner ${ }^{3}$, Klaus Müllen Nigel T. Lucas ${ }^{* 1}$, Michael W. George ${ }^{2,5 *}$ and Keith C. Gordon ${ }^{1 *}$

${ }^{1}$ MacDiarmid Institute for Advanced Materials and Nanotechnology and Department of Chemistry, University of Otago, Dunedin, New Zealand

${ }^{2}$ Department of Chemistry, University of Nottingham, Nottingham, United Kingdom

${ }^{3}$ School of Chemistry, University of Tasmania, Private Bag 75, Hobart 7001, Australia

${ }^{4}$ Max Planck Institute for Polymer Research, Ackermannweg 10, Mainz 55128, Germany

${ }^{5}$ Department of Chemical and Environmental Engineering, University of Nottingham Ningbo China, 199 Talking East Road, Ningbo 315100, China

\section{SUPPORTING INFORMATION}

Synthesis

NMR and MS Data

Solid-state Structural Characterization \& Packing Detail

Calculations

Spectroscopic Data

References 
Scheme S1. Synthesis of bpy-HBC $(\mathrm{R})_{5}$ ligands (3) and their $\operatorname{Re}(\mathrm{CO})_{3} \mathrm{Cl}$ complexes $(4){ }^{a}$
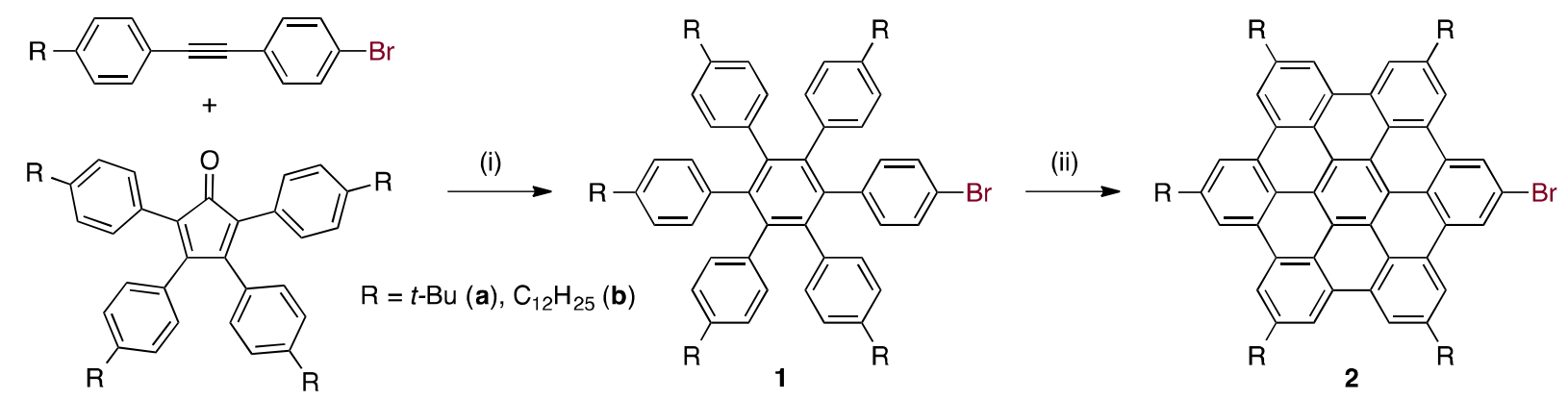

(iii)
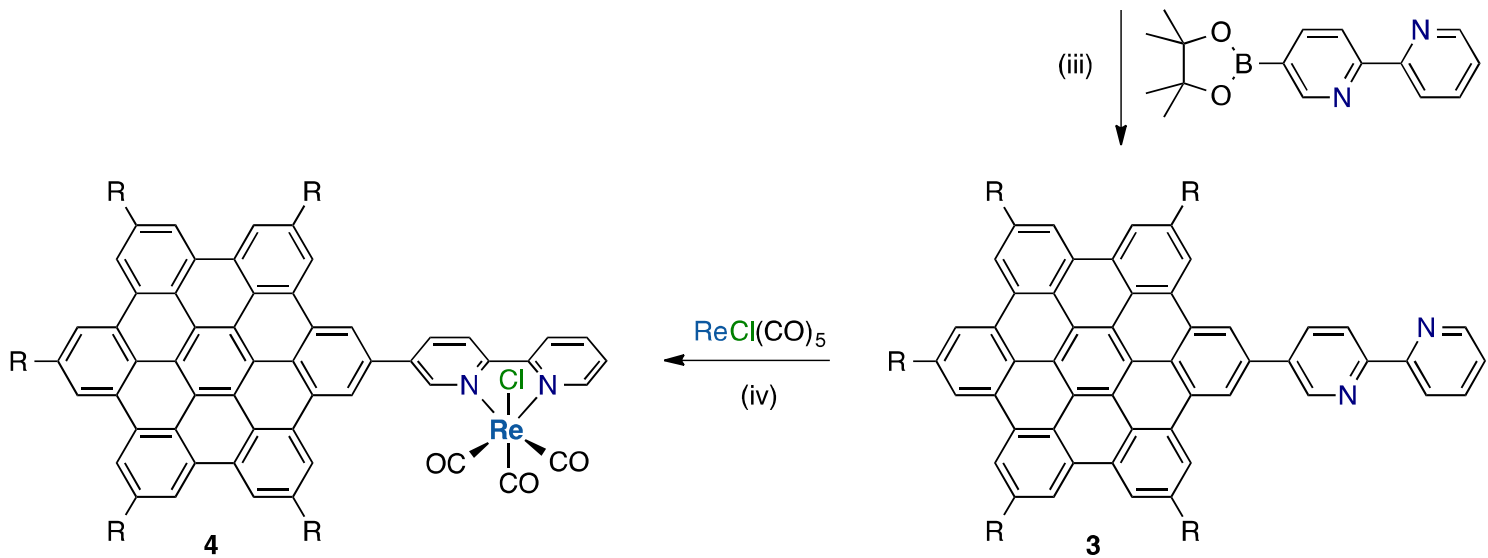

${ }^{a}$ (i) diphenyl ether, reflux, 18 h; (ii) $\mathrm{FeCl}_{3} / \mathrm{CH}_{3} \mathrm{NO}_{2}, \mathrm{DCM}$, rt, 20 min; (iii) $\mathrm{Pd}\left(\mathrm{PPh}_{3}\right)_{4}, \mathrm{~K}_{2} \mathrm{CO}_{3}$, toluene, water, 1-propanol, 85 ${ }^{\circ} \mathrm{C}$, 18-20 h; (iv) toluene, reflux, $5 \mathrm{~h}$.

General conditions and materials. The reactions were carried out under an inert atmosphere using standard Schlenk techniques. Tetra(4-tert-butylphenyl)cyclopentadienone, ${ }^{1} \quad$ 2-bromo-5,8,11,14,17-penta(dodecyl)hexa-peri-hexabenzocoronene $(\mathbf{2 b})^{2}$ and 5-(4,4,5,5-tetramethyl-[1,3,2]dioxaborolan-2-yl)-2,2'-bipyridine ${ }^{3}$ were synthesised according to literature procedures (or minor variations thereof). All other chemicals were commercially purchased and used as received unless otherwise stated. Dry solvents were obtained from a Pure-Solv MD-6 Solvent Purification system; all other solvents were AR grade. ${ }^{1} \mathrm{H}$ NMR ( $\left.500 \mathrm{MHz}\right)$ and ${ }^{13} \mathrm{C}$ NMR $(126 \mathrm{MHz})$ spectra were recorded on a Varian 500 AR spectrometer at 25 ${ }^{\circ} \mathrm{C}$, and are referenced to residual $\mathrm{CHCl}_{3}(7.26 \mathrm{ppm})$ and $\mathrm{CDCl}_{3}(77.16 \mathrm{ppm})$, respectively. ESI mass spectra were recorded on a Bruker MicrOTOF-Q mass spectrometer. ${ }^{1} \mathrm{H}$ and ${ }^{13} \mathrm{C}$ NMR spectra were assigned using $2 \mathrm{D}$ spectroscopies (COSY, NOESY, ${ }^{1} \mathrm{H},{ }^{13} \mathrm{C}-\mathrm{HSQC}$ and $\left.{ }^{1} \mathrm{H},{ }^{13} \mathrm{C}-\mathrm{HMBC}\right)$. MALDI-TOF mass spectra were recorded on an Applied Biosystems 4800 Tandem TOF mass spectrometer with external calibration to within $m / z \pm 0.08$; solid analyte and TCNQ matrix were mixed using a mini mixer-mill, suspended in hexane and transferred to the sample plate. Microanalyses were performed at the Campbell Microanalytical Laboratory, University of Otago, Dunedin, New Zealand.

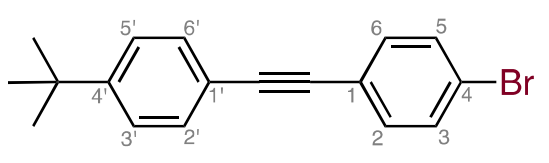

(4-Bromophenyl)(4'-tert-butylphenyl)ethyne. A solution of 4bromoiodobenzene $(4.68 \mathrm{~g}, 16.5 \mathrm{mmol})$ in triethylamine $(8 \mathrm{o} \mathrm{mL})$ was bubbled with argon for $10 \mathrm{~min}$, then 4-tert-butylphenylethyne (3.00 $\mathrm{mL}, 2.63 \mathrm{~g}, 16.6 \mathrm{mmol})$ added and the mixture bubbled with argon for a further $5 \mathrm{~min}$. CuI $(65 \mathrm{mg}, 0.34$ $\mathrm{mmol})$ and $\mathrm{PdCl}_{2}\left(\mathrm{PPh}_{3}\right)_{2}(230 \mathrm{mg}, 0.33 \mathrm{mmol})$ were added and the reaction mixture stirred under argon at rt for $2 \mathrm{~h}$, during which a light-grey precipitate formed. The mixture was filtered, the flask and precipitate washed with hexane $(2 \times 20$ $\mathrm{mL})$, and the filtrate taken to dryness in vacuo. The residue was purified by column chromatography $\left(\mathrm{SiO}_{2}\right.$, hexane) to afford (4-bromophenyl)(4'-tert-butylphenyl)ethyne as a pale yellow powder (4.91 g, $15.7 \mathrm{mmol}, 95 \%)$. ${ }^{1} \mathrm{H}$ NMR $(500 \mathrm{MHz}$, $\left.\mathrm{CDCl}_{3}\right): \delta 7.47\left(\mathrm{~d}, J=8.5 \mathrm{~Hz}, 2 \mathrm{H}, H_{3,5}\right), 7.45\left(\mathrm{~d}, J=8.2 \mathrm{~Hz}, 2 \mathrm{H}, H_{2^{\prime}, 6^{\prime}}\right), 7.38\left(\mathrm{~d}, J=8.8 \mathrm{~Hz}, 2 \mathrm{H}, H_{2,6}\right), 7.37(\mathrm{~d}, J=8.8 \mathrm{~Hz}, 2 \mathrm{H}$, $\left.H_{3^{\prime}, 5^{\prime}}\right), 1.33\left(\mathrm{~s}, 9 \mathrm{H}, \mathrm{C}\left(\mathrm{CH}_{3}\right)_{3}\right)$ ppm. ${ }^{13} \mathrm{C}$ NMR $\left(126 \mathrm{MHz}, \mathrm{CDCl}_{3}\right): \delta 152.00\left(C_{4^{\prime}}\right), 133.14\left(C_{2,6}\right), 131.72\left(C_{3,5}\right), 131.48\left(C_{2^{\prime}, 6^{\prime}}\right), 125.56$ $\left(C_{3^{\prime}, 5^{\prime}}\right), 122.66\left(C_{1}\right), 122.38\left(C_{4}\right), 120.02\left(C_{1^{\prime}}\right)$, $90.84\left(\mathrm{C} \equiv C^{\prime}\right), 87.82\left(C \equiv C^{\prime}\right)$, 34.98 $\left(C\left(\mathrm{CH}_{3}\right)_{3}\right), 31.31\left(\mathrm{C}\left(\mathrm{CH}_{3}\right)_{3}\right)$ ppm. MALDI-TOF 
MS: Calcd for $[\mathrm{M}]^{+}\left(\mathrm{C}_{18} \mathrm{H}_{17} \mathrm{Br}\right): \mathrm{m} / \mathrm{z}$ (\%) 312.05 (100), 313.05 (20), 314.05 (99), 315.05 (19); found: 312.06 (98), 313.04 (35), 314.07 (100), 315.07 (27). Anal. calcd for $\mathrm{C}_{18} \mathrm{H}_{17} \mathrm{Br}$ : C, 69.02; H, 5.47. Found: C, 69.27; H, 5.63.

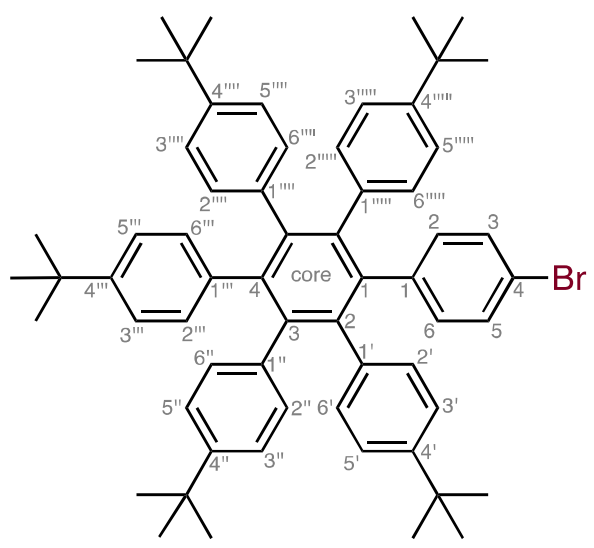

4-Bromo-4',4",4"',4"'",4"'"'-penta(tert-butyl)hexaphenylbenzene (1a). A purple solution of tetra(4-tert-butylphenyl)cyclopentadienone $(1.25 \mathrm{~g}, 2.06$ $\mathrm{mmol}$ ) and (4-bromophenyl)(4'-tert-butylphenyl)ethyne (o.682 g, $2.19 \mathrm{mmol})$ dissolved in diphenyl ether (10 $\mathrm{mL}$ ) was bubbled with argon for $10 \mathrm{~min}$ and then heated at a gentle reflux $\left(\mathrm{ca} .230^{\circ} \mathrm{C}\right)$ for $18 \mathrm{~h}$. After cooling to rt, ethanol was added and the light-brown reaction mixture filtered to give crude $\mathbf{1 a}$ as an off-white powder. Further purification by column chromatography $\left(\mathrm{SiO}_{2}\right.$, hexane/dichloromethane) afforded $1 \mathrm{a}$ as a white powder (1.14 g, $1.28 \mathrm{mmol}$, 62\%). ${ }^{1} \mathrm{H}$ NMR (50o MHz, $\left.\mathrm{CDCl}_{3}\right): \delta 6.96\left(\mathrm{~d}, J=8.5 \mathrm{~Hz}, 2 \mathrm{H}, H_{3,5}\right), 6.85(\mathrm{~d}, J=$ 8.o $\mathrm{Hz}, 4 \mathrm{H}, H_{\left.3^{\prime} 5^{\prime}, 3^{\prime \prime \prime \prime}, 5^{\prime \prime \prime \prime}\right), 6.81}\left(\mathrm{~d}, J=8.5 \mathrm{~Hz}, 2 \mathrm{H}, H_{\left.3^{\prime \prime \prime}, 5^{\prime \prime \prime}\right), 6.79}(\mathrm{~d}, J=8.5 \mathrm{~Hz}, 4 \mathrm{H}\right.$, $H_{\left.3^{\prime \prime}, 5^{\prime \prime}, 3^{\prime \prime \prime}, 5^{\prime \prime \prime \prime}\right), 6.71}\left(\mathrm{~d}, J=8.5 \mathrm{~Hz}, 2 \mathrm{H}, H_{2,6}\right), 6.67(\mathrm{~d}, J=8.0 \mathrm{~Hz}, 6 \mathrm{H}$,

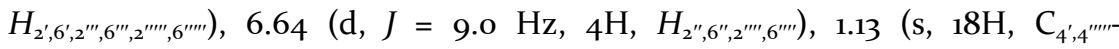

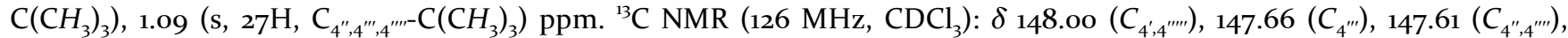
$141.00\left(C_{\text {core- }-4}\right), 140.89\left(C_{\text {core-3,5 }}\right)$, 140.32 $\left(C_{1}\right)$, $140.22\left(C_{\text {core- }-6,6}\right), 138.62\left(C_{\text {core- } 1}\right)$, $137.90\left(C_{1^{\prime \prime \prime}}\right), 137.87\left(C_{1^{\prime \prime}, 1^{\prime \prime \prime}}\right), 137.69\left(C_{1^{\prime}, 1^{\prime \prime \prime \prime}}\right), 133.44$

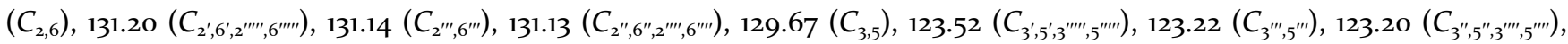

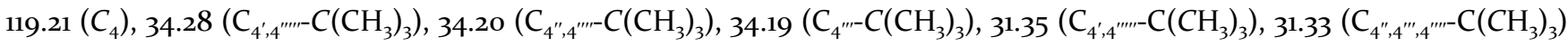
ppm. MALDI-TOF MS: Calcd for [M] ${ }^{+}\left(\mathrm{C}_{62} \mathrm{H}_{69} \mathrm{Br}\right): \mathrm{m} / \mathrm{z}$ (\%) 892.46 (83), 893.46 (57), 894.46 (100), 895.46 (59), 896.46 (19), 897.47 (4); found: 892.42 (86), 893.44 (64), 894.42 (100), 895.43 (59), 896.43 (22), 897.43 (5). Anal. calcd for $\mathrm{C}_{62} \mathrm{H}_{69} \mathrm{Br}_{\mathrm{C}} \mathrm{C}$, 83.28; H, 7.78. Found: C, 83.49; H, 8.02.

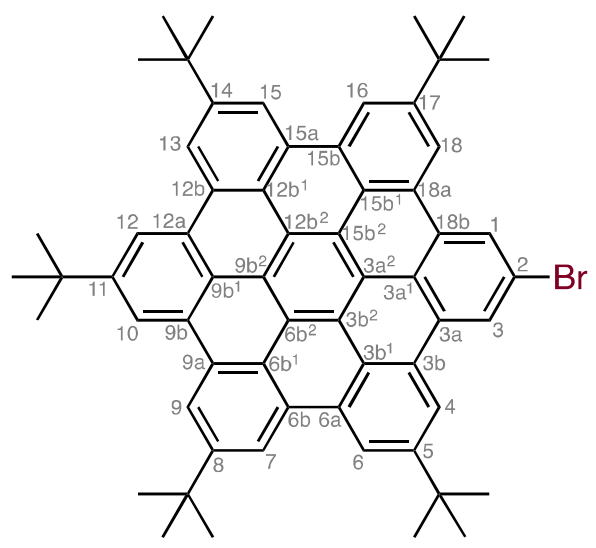

2-Bromo-5,8,11,14,17-penta(tert-butyl)hexa-peri-hexabenzocoronene (2a). A solution of hexaphenylbenzene $1 \mathbf{a}(0.570 \mathrm{~g}, 0.638 \mathrm{mmol})$ in dry dichloromethane $(160 \mathrm{~mL})$ was bubbled with nitrogen for $10 \mathrm{~min}$, then a solution of $\mathrm{FeCl}_{3}(2.42 \mathrm{~g}, 14.9 \mathrm{mmol})$ in nitromethane $(15 \mathrm{~mL})$ was added dropwise. The dark mixture was stirred for 20 min while gentle bubbling with nitrogen was continued. The reaction was quenched with methanol $(5 \mathrm{~mL})$ causing the color to change to yellow. The organic phase was washed with water until no yellow coloration of the aqueous phase was observed, then dried $\left(\mathrm{MgSO}_{4}\right)$, filtered, and the solvent removed. The residue was purified by column chromatography $\left(\mathrm{SiO}_{2}\right.$, dichloromethane) to give $\mathbf{2 a}$ as a yellow powder (o.548 $\mathrm{g}$, $0.621 \mathrm{mmol}, 97 \%)$. ${ }^{1} \mathrm{H}$ NMR (500 MHz, $\left.\mathrm{CDCl}_{3} / \mathrm{CS}_{2}(1: 1), 6 \mathrm{mM}\right): \delta 9.24(\mathrm{~s}, 2 \mathrm{H}$, $\left.H_{10,12}\right), 9.21\left(\mathrm{~d}, J=1.5 \mathrm{~Hz}, 2 \mathrm{H}, H_{9,13}\right), 9.17\left(\mathrm{~s}, 2 \mathrm{H}, H_{7,15}\right), 9.15\left(\mathrm{~s}, 2 \mathrm{H}, H_{6,16}\right), 9.02(\mathrm{~s}$, $\left.2 \mathrm{H}, \mathrm{H}_{1,3}\right), 8.96\left(\mathrm{~s}, 2 \mathrm{H}, \mathrm{H}_{4,18}\right), 1.88\left(\mathrm{~s}, 9 \mathrm{H}, \mathrm{C}_{11}-\mathrm{C}\left(\mathrm{CH}_{3}\right)_{3}\right), 1.87\left(\mathrm{~s}, 18 \mathrm{H}, \mathrm{C}_{8,14}-\mathrm{C}\left(\mathrm{CH}_{3}\right)_{3}\right), 1.83\left(\mathrm{~s}, 18 \mathrm{H}, \mathrm{C}_{5,17}-\mathrm{C}\left(\mathrm{CH}_{3}\right)_{3}\right) \mathrm{ppm} .{ }^{13} \mathrm{C} \mathrm{NMR}$ $\left(126 \mathrm{MHz}, \mathrm{CDCl}_{3} / \mathrm{CS}_{2}(1: 1), 6 \mathrm{mM}\right): \delta 148.75\left(C_{11}\right), 148.74\left(C_{5,17}\right), 148.65\left(C_{8,14}\right), 132.46\left(C_{3 \mathrm{a}, 18 \mathrm{~b}}\right), 130.47\left(C_{3 \mathrm{~b} 2,15 \mathrm{~b} 2}\right), 130.35\left(C_{9 \mathrm{a}, 1 \mathrm{~b}}\right)$, $130.21\left(C_{6 \mathrm{a}, 6 \mathrm{~b}, 15 \mathrm{a}, 15 \mathrm{~b}}\right), 128.89\left(C_{3 \mathrm{~b}, 18 \mathrm{a}}\right), 124.16\left(C_{1,3}\right), 123.7 \mathrm{O}\left(C_{3 \mathrm{c}, 15 \mathrm{c}}\right), 123.65\left(C_{6 \mathrm{c}, 9 \mathrm{c}, 12 \mathrm{c}}\right), 121.77\left(C_{18 \mathrm{c}}\right), 120.67\left(C_{9 \mathrm{bz} 2}\right), 120.39\left(C_{6 \mathrm{bz}, 12 \mathrm{ba} 2}\right)$, $120.19\left(C_{3 \mathrm{~b} 2,15 \mathrm{b2}}\right), 119.56\left(C_{3 \mathrm{az}}\right), 119.41\left(C_{6,16}\right), 119.29\left(C_{4,18}\right), 118.91\left(C_{7,9,10,12,13,15}\right), 35.66\left(\mathrm{C}_{11}-C\left(\mathrm{CH}_{3}\right)_{3}\right), 35.62\left(\mathrm{C}_{8,14}-C\left(\mathrm{CH}_{3}\right)_{3}\right), 35.61$ $\left(\mathrm{C}_{5,17}-\mathrm{C}\left(\mathrm{CH}_{3}\right)_{3}\right)$, $32.09\left(\mathrm{C}_{11}-\mathrm{C}\left(\mathrm{CH}_{3}\right)_{3}\right), 32.08\left(\mathrm{C}_{8,14}-\mathrm{C}\left(\mathrm{CH}_{3}\right)_{3}\right)$, $32.05\left(\mathrm{C}_{5,17}-\mathrm{C}\left(\mathrm{CH}_{3}\right)_{3}\right)$ ppm; the $\mathrm{C}_{2}-\mathrm{Br}$ signal was not observed. MALDI-TOF MS: Calcd for $[\mathrm{M}]^{+}\left(\mathrm{C}_{62} \mathrm{H}_{57} \mathrm{Br}\right): \mathrm{m} / \mathrm{z}$ (\%) 880.36 (83), 881.37 (56), 882.36 (100), 883.37 (59), 884.37 (19), 885.37 (4); found: 880.36 (89), 881.37 (64), 882.36 (100), 883.37 (64), 884.37 (24), 885.37 (5). Anal. calcd for $\mathrm{C}_{62} \mathrm{H}_{57} \mathrm{Br}^{\circ} \cdot 0.1\left(\mathrm{CH}_{2} \mathrm{Cl}_{2}\right)$ : C, 83.76; H, 6.47. Found: C, 83.56; H, 6.52. 
${ }^{1} \mathrm{H} \mathrm{NMR}\left(\mathrm{CDCl}_{3}, 500 \mathrm{MHz}, 298 \mathrm{~K}\right)$
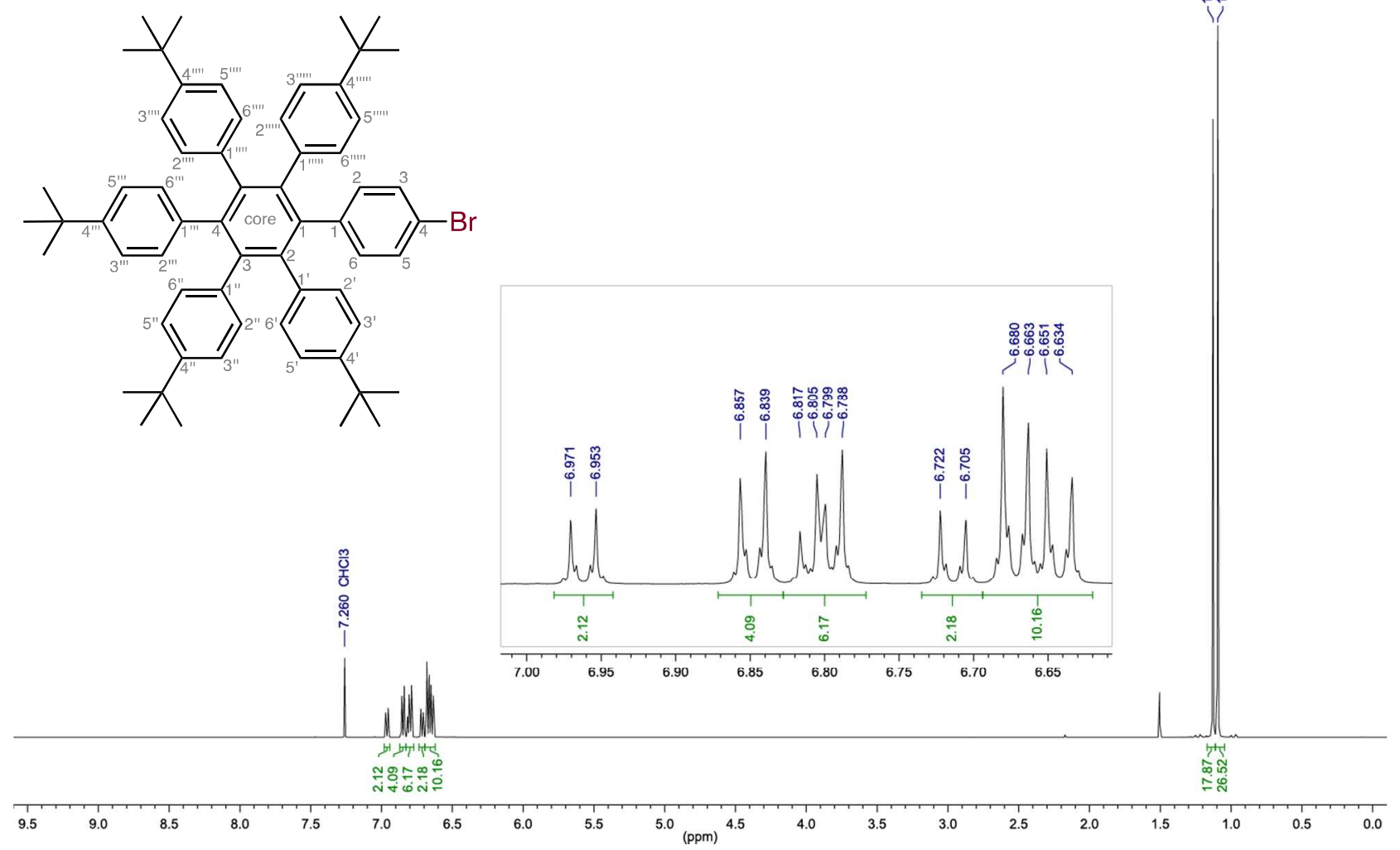

${ }^{13} \mathrm{C} \mathrm{NMR}\left(\mathrm{CDCl}_{3}, 126 \mathrm{MHz}, 298 \mathrm{~K}\right)$
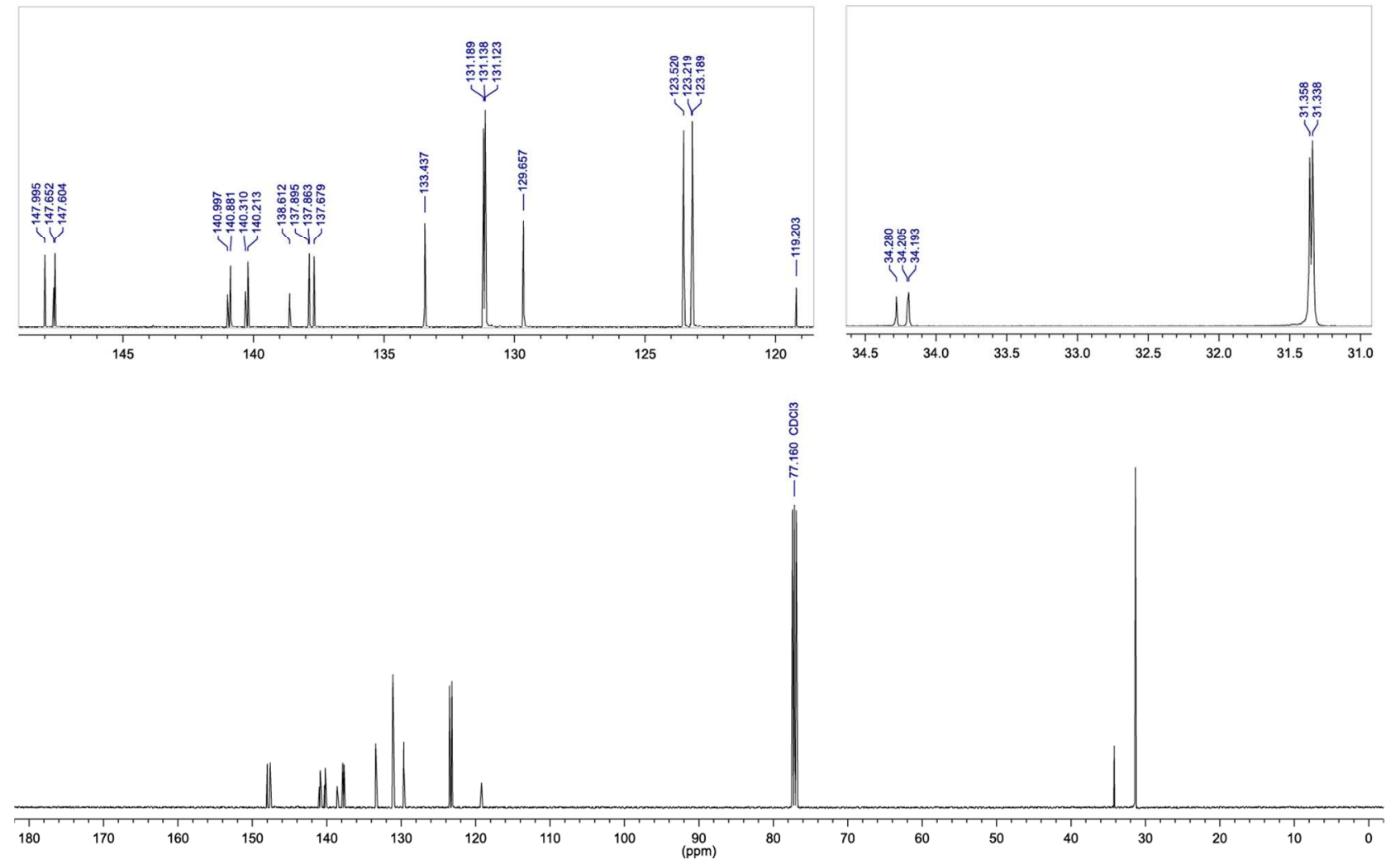


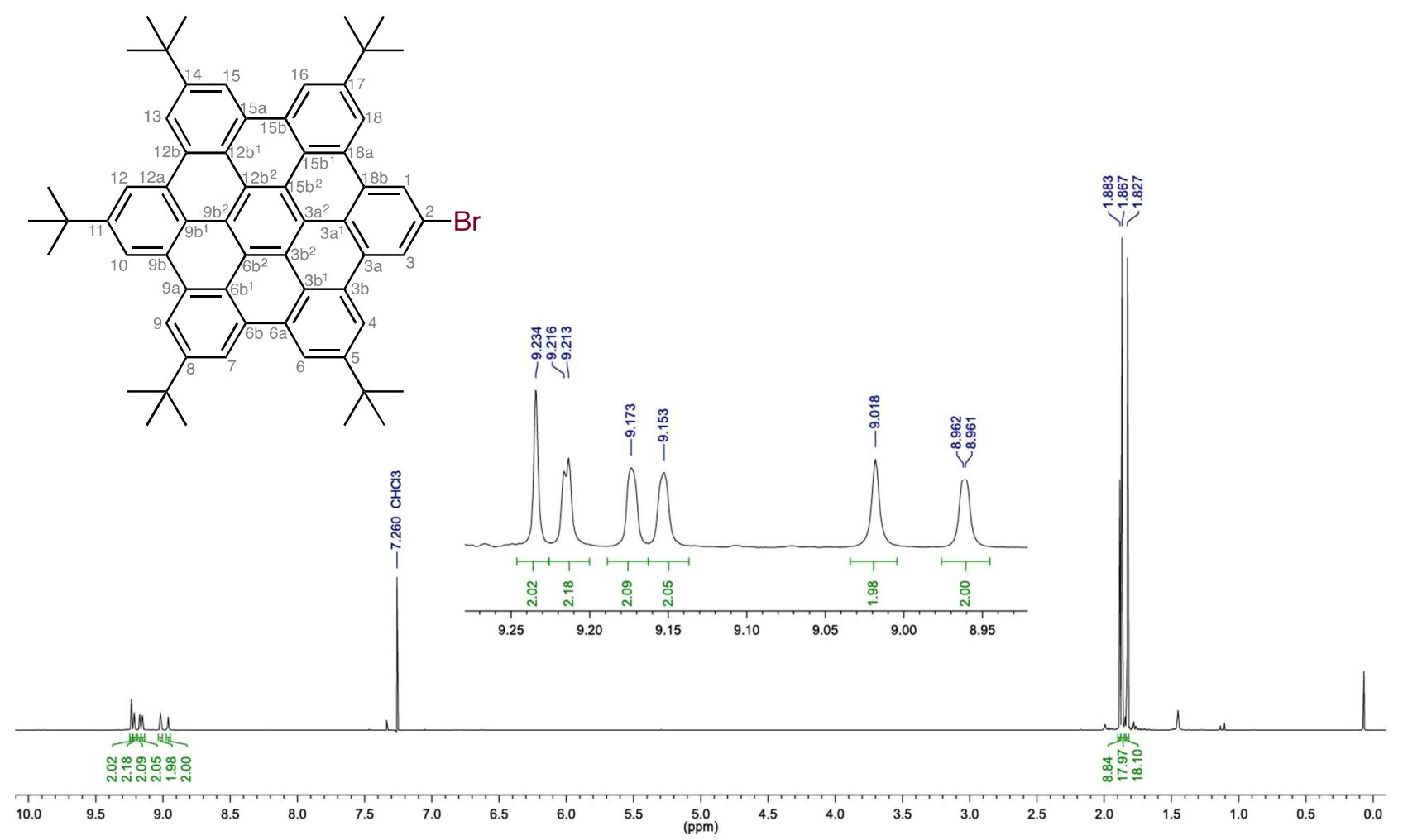

${ }^{13} \mathrm{C} \mathrm{NMR}\left(\mathrm{CDCl}_{3} / \mathrm{CS}_{2}(1: 1), 126 \mathrm{MHz}, 298 \mathrm{~K}\right)$

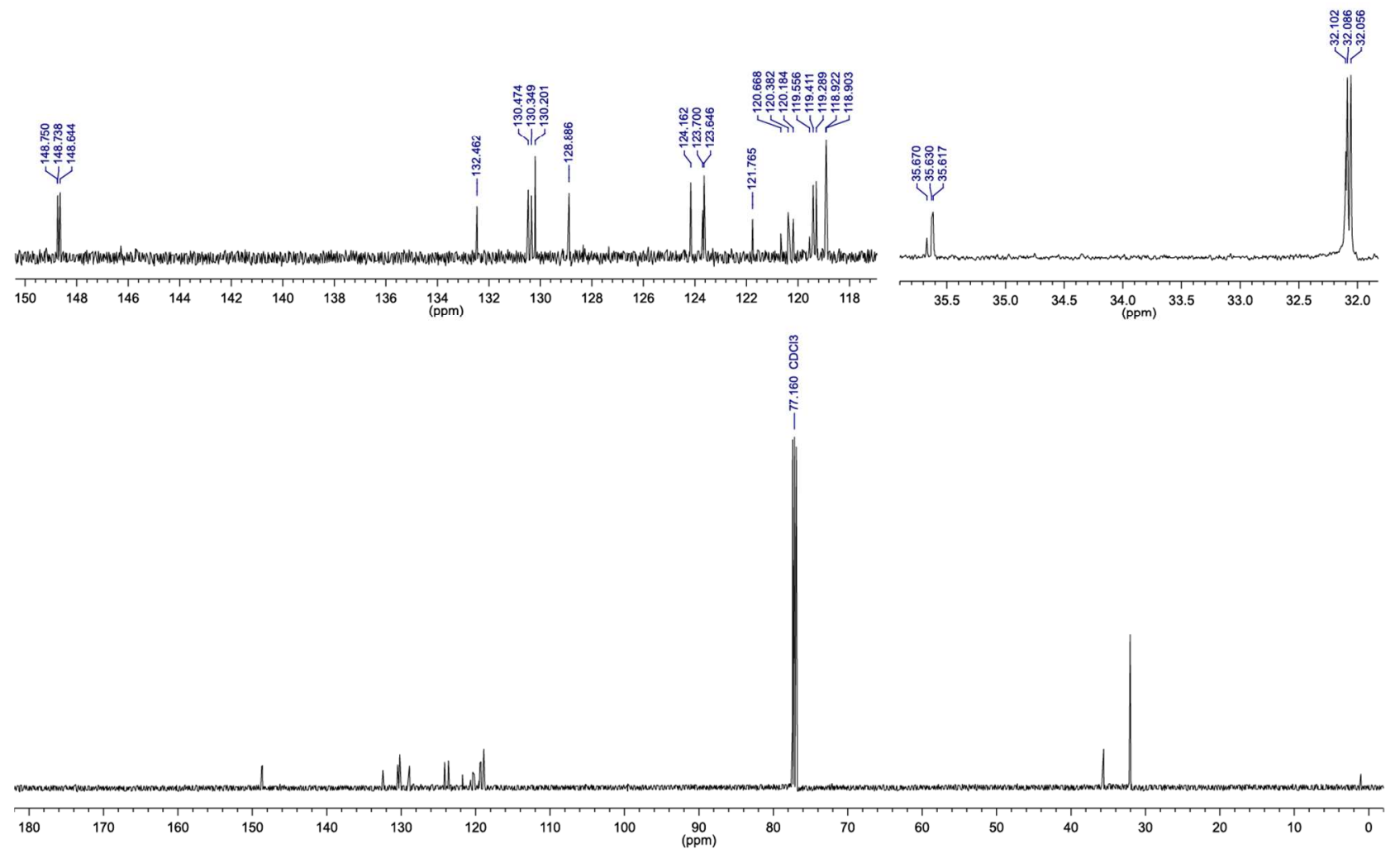


${ }^{1} \mathrm{H} \mathrm{NMR}\left(\mathrm{CDCl}_{3} / \mathrm{CS}_{2}(1: 1), 500 \mathrm{MHz}, 298 \mathrm{~K}\right)$

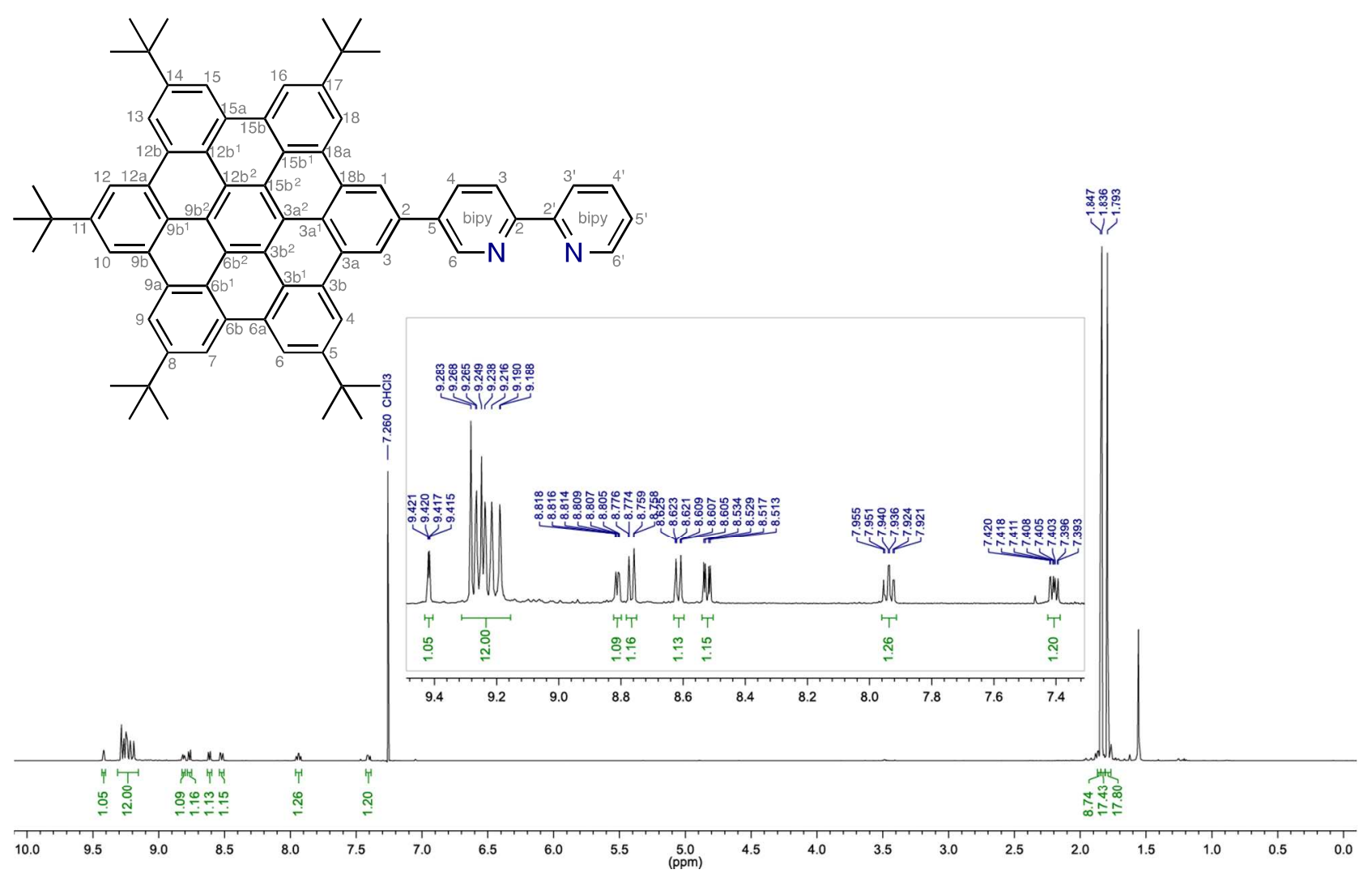

${ }^{13} \mathrm{C} \mathrm{NMR}\left(\mathrm{CDCl}_{3}, 126 \mathrm{MHz}, 298 \mathrm{~K}\right)$

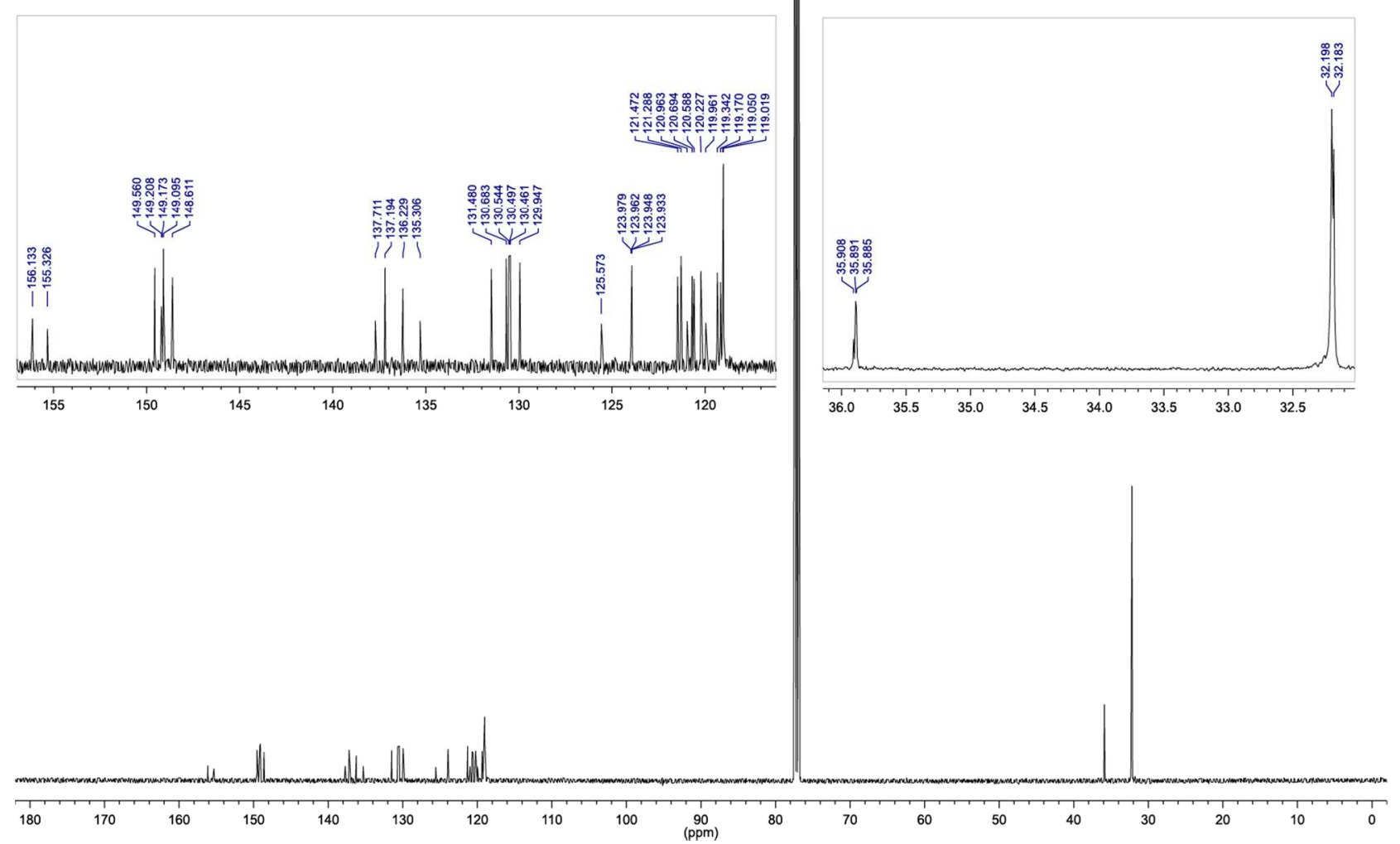




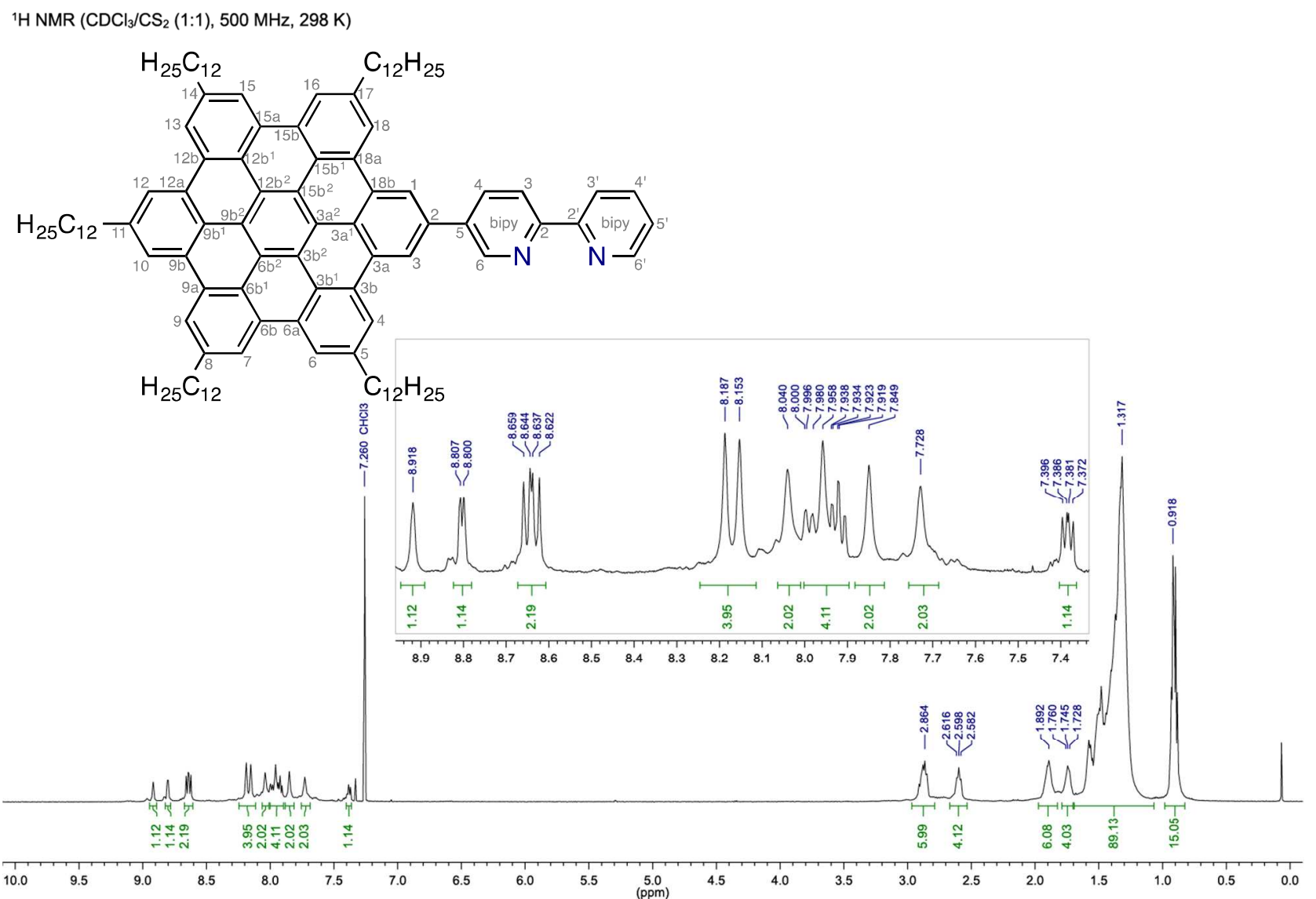

${ }^{13} \mathrm{C} \mathrm{NMR}\left(\mathrm{CDCl}_{3} / \mathrm{CS}_{2}(1: 1), 126 \mathrm{MHz}, 298 \mathrm{~K}\right)$
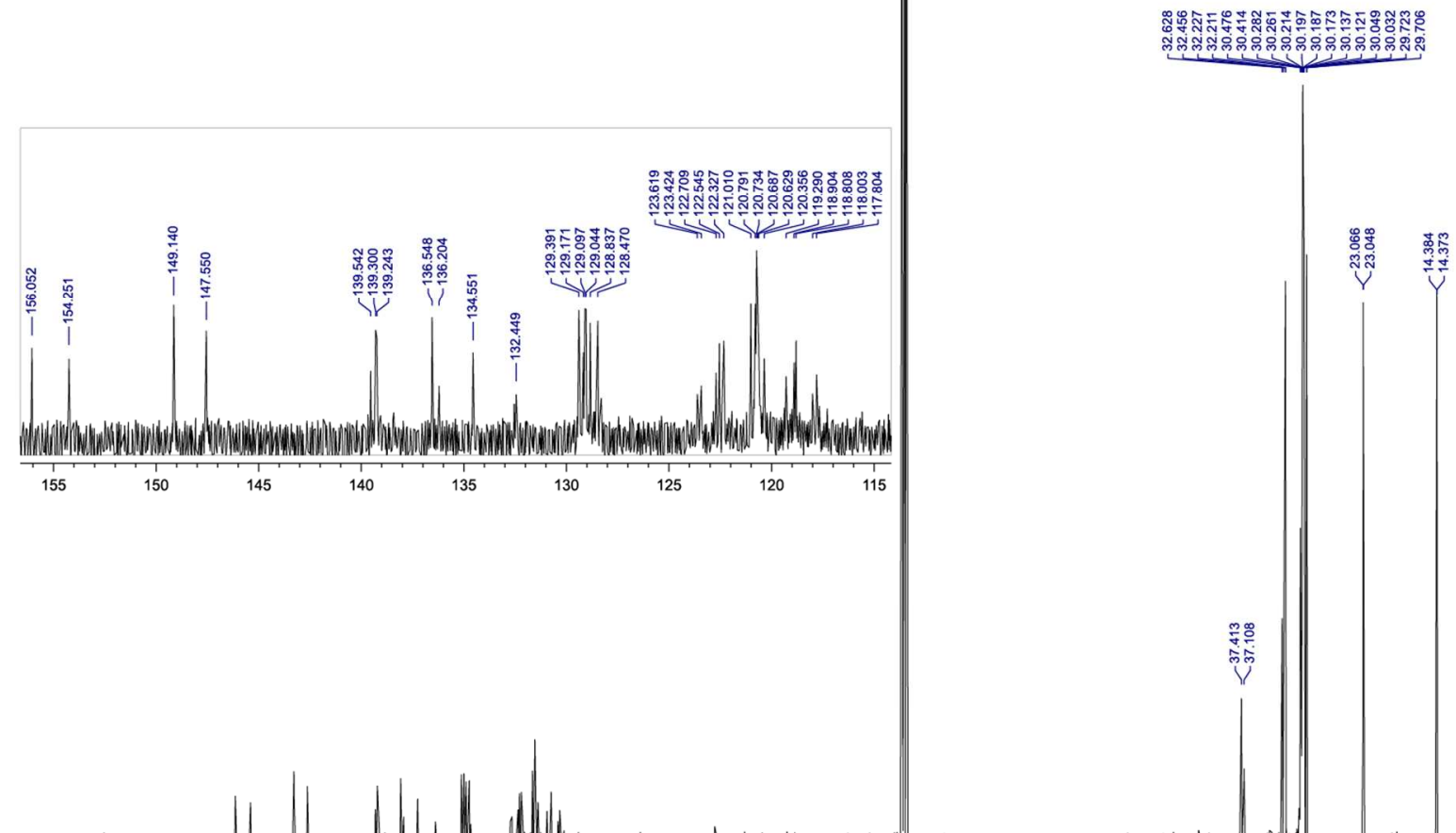

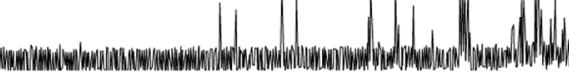




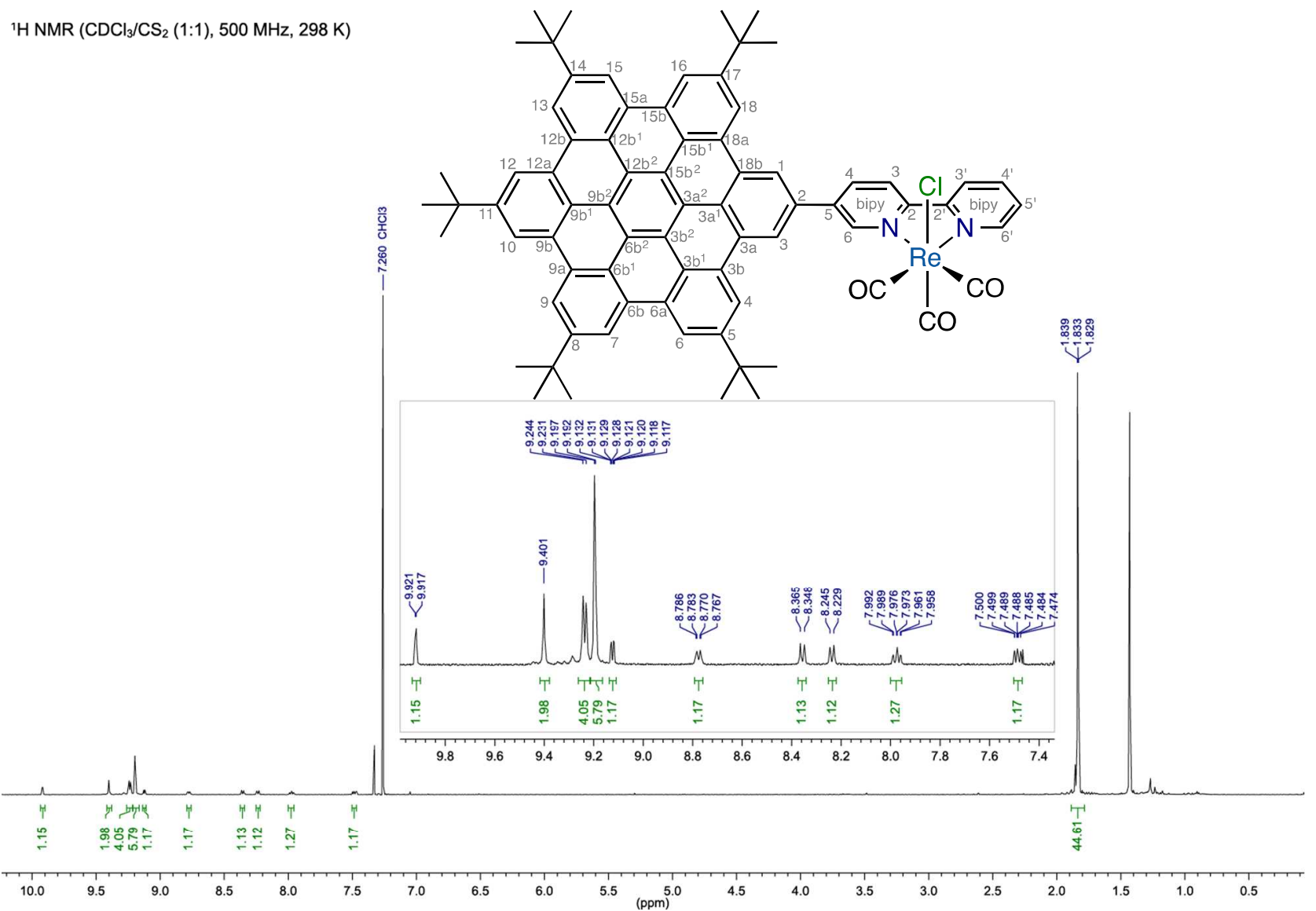

${ }^{13} \mathrm{C} \mathrm{NMR}\left(\mathrm{CDCl}_{3} / \mathrm{CS}_{2}(1: 1), 126 \mathrm{MHz}, 298 \mathrm{~K}\right)$
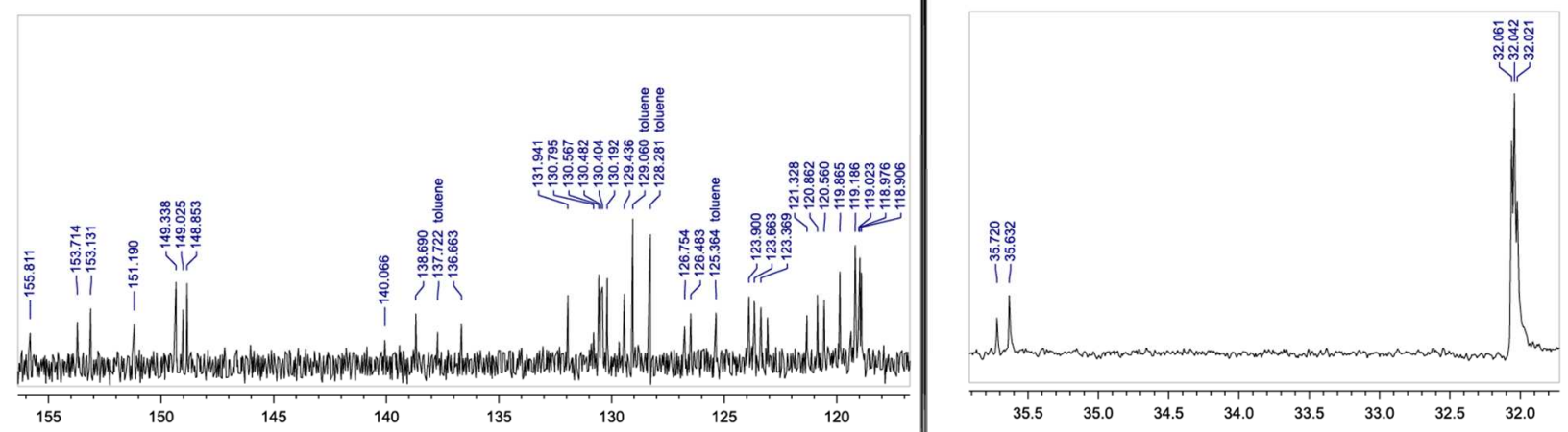

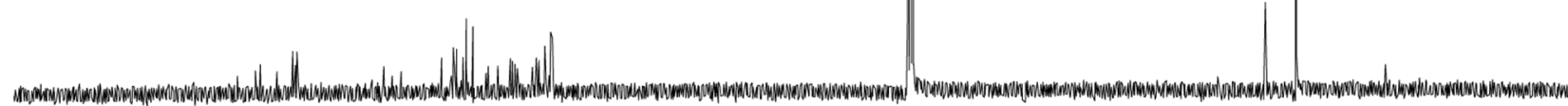

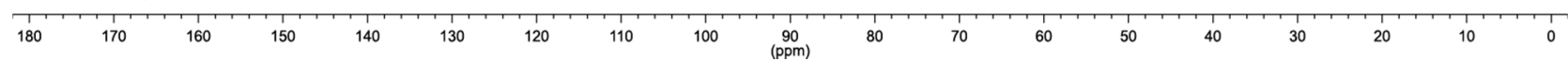


${ }^{1} \mathrm{H} \mathrm{NMR}\left(\mathrm{CDCl}_{3} / \mathrm{CS}_{2}(1: 1), 500 \mathrm{MHz}, 298 \mathrm{~K}\right)$

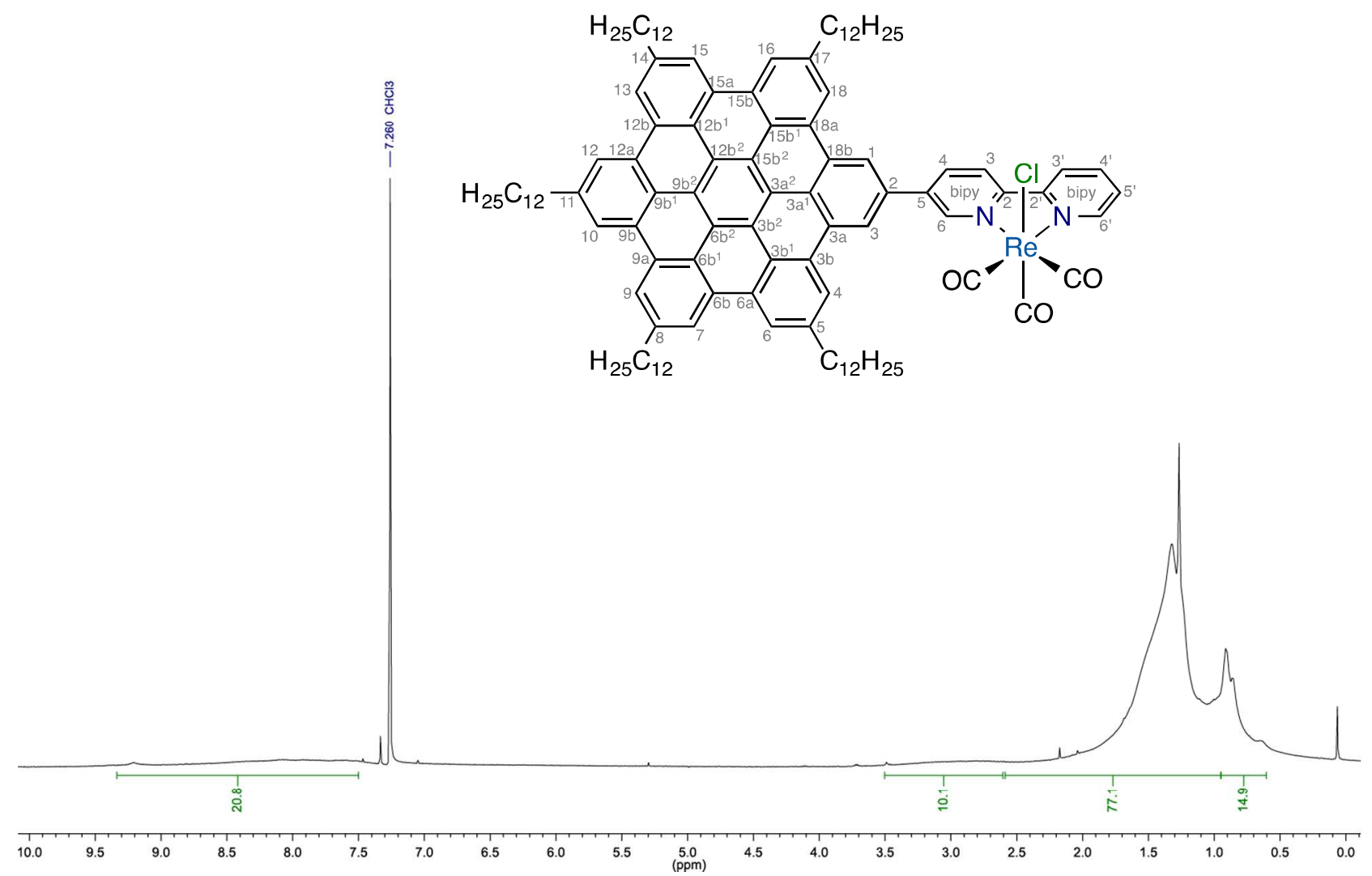

${ }^{13} \mathrm{C} \mathrm{NMR}\left(\mathrm{CDCl}_{3} / \mathrm{CS}_{2}(1: 1), 126 \mathrm{MHz}, 298 \mathrm{~K}\right)$

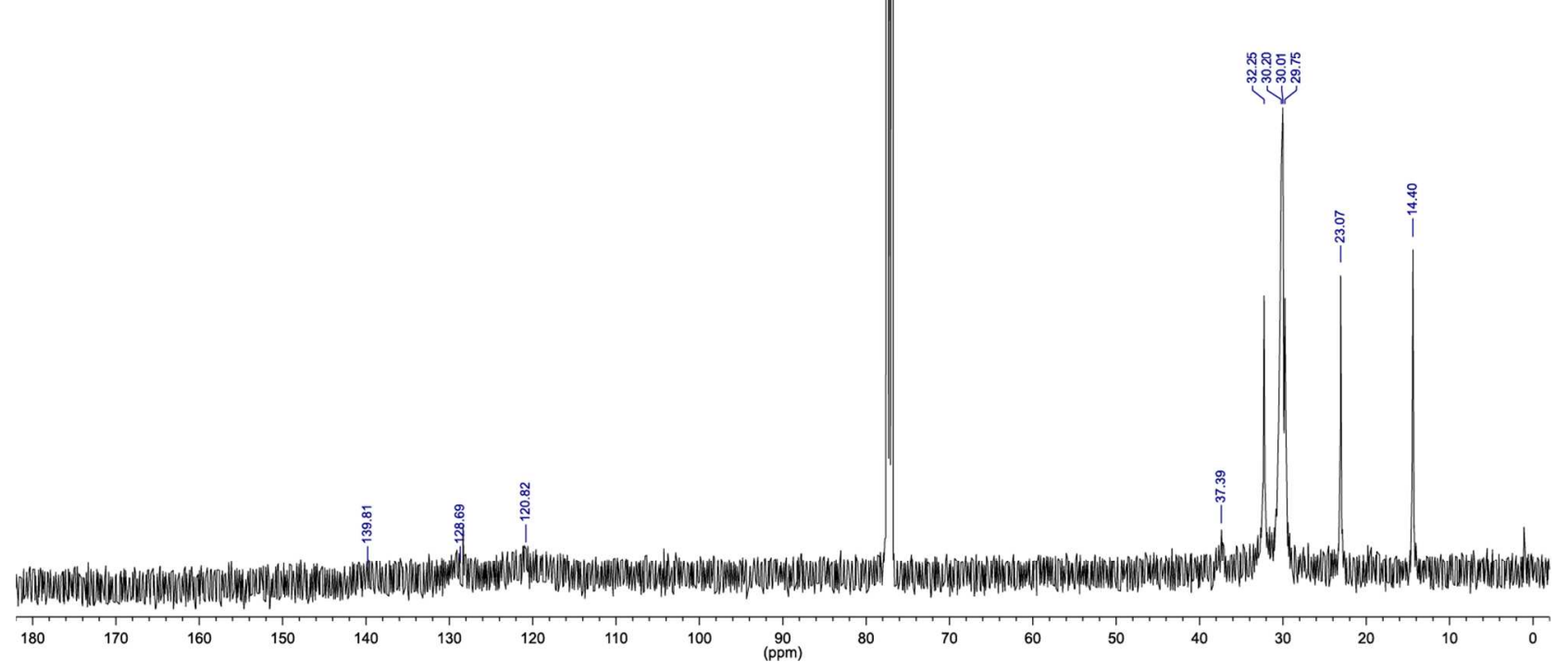




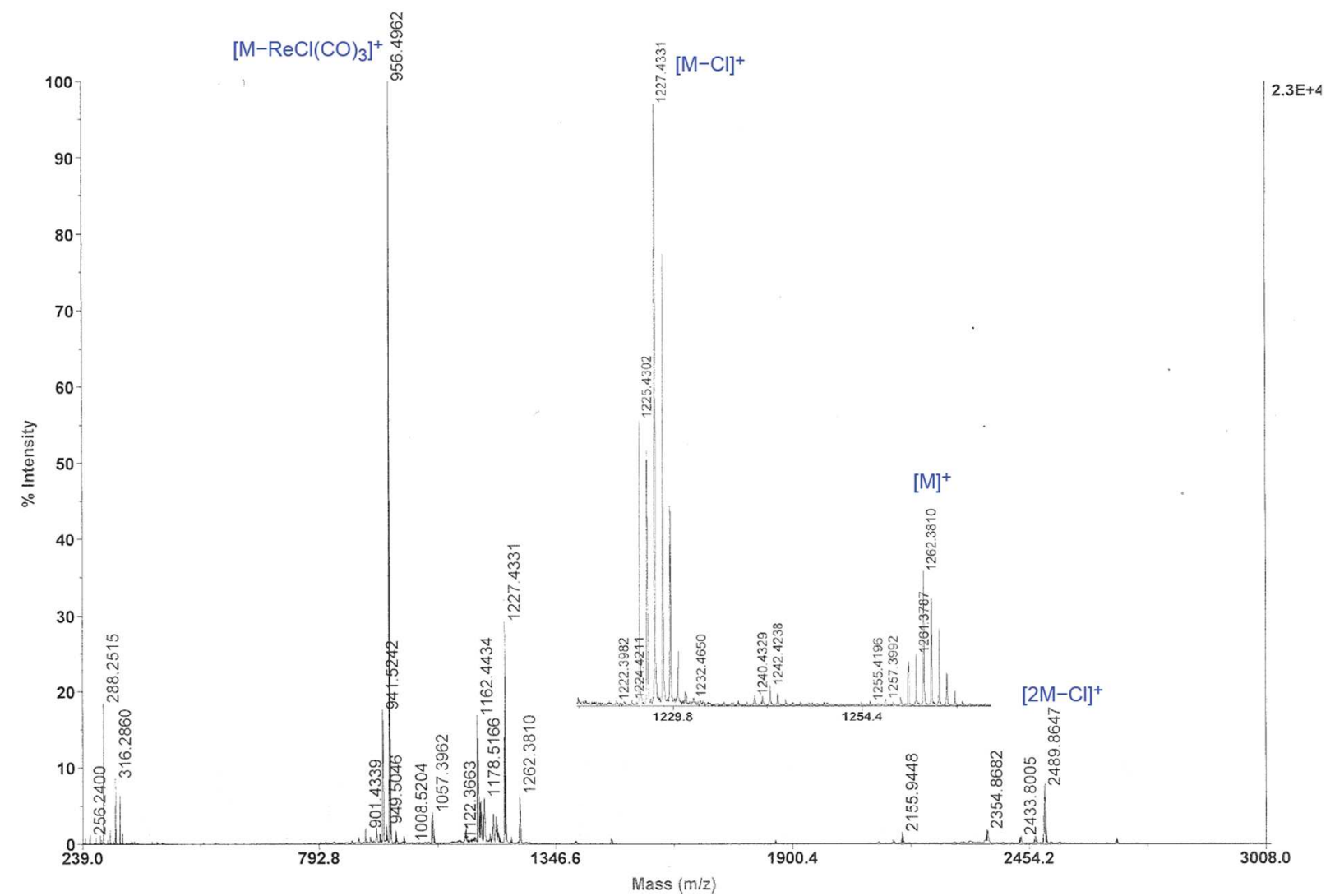

MALDI-TOF spectrum (TCNQ matrix) for $\mathbf{4 a}$

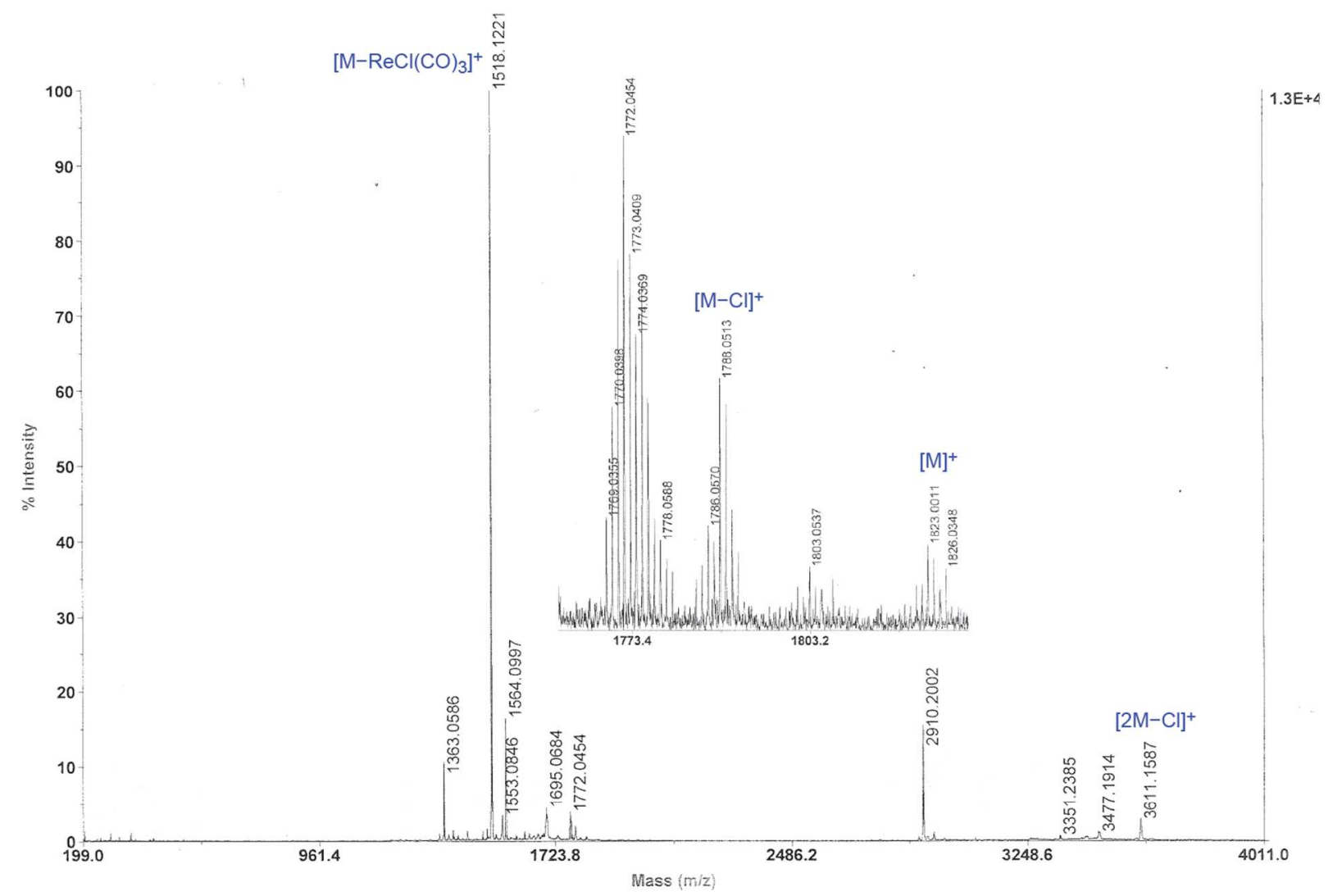

MALDI-TOF spectrum (TCNQ matrix) for $\mathbf{4 b}$ 


\section{Solid-state Structural Characterization \& Packing Detail}

Analysis of the HBC bond lengths reveals the six outermost benzenoid rings and the central benzenoid ring to have an average C-C bond length of 1.404(9) $\AA$, while the other twelve C-C bonds are longer with an average length of 1.455(8) $\AA$, consistent with Clar's aromatic sextet description of HBCs. ${ }^{4}$

The HBCs pack as head-to-tail inversion-related dimers, offset so as to minimise steric interactions involving the tertbutyl groups and allowing a close stacking approach of the aromatic cores (Figure $\mathrm{S}$ ). The chloride ligands point to the outside of the dimer pair while the carbonyl ligand trans to the chloride participates in a number of hydrogen-bonding interactions with the adjacent HBC core and tert-butyl groups, strengthening the supramolecular association. Furthermore, the crystal structure shows that the $\mathbf{4}$ a complexes are arranged with an offset face-to-face arrangement at the bipyridine end of the molecule, again across an inversion center (Figure S2); this stacking arrangement is reinforced by a number of hydrogen-bonding interactions between the chloride ligand and hydrogen atoms from the neighboring bipyridine, tert-butyl group and a chloroform solvate molecule.

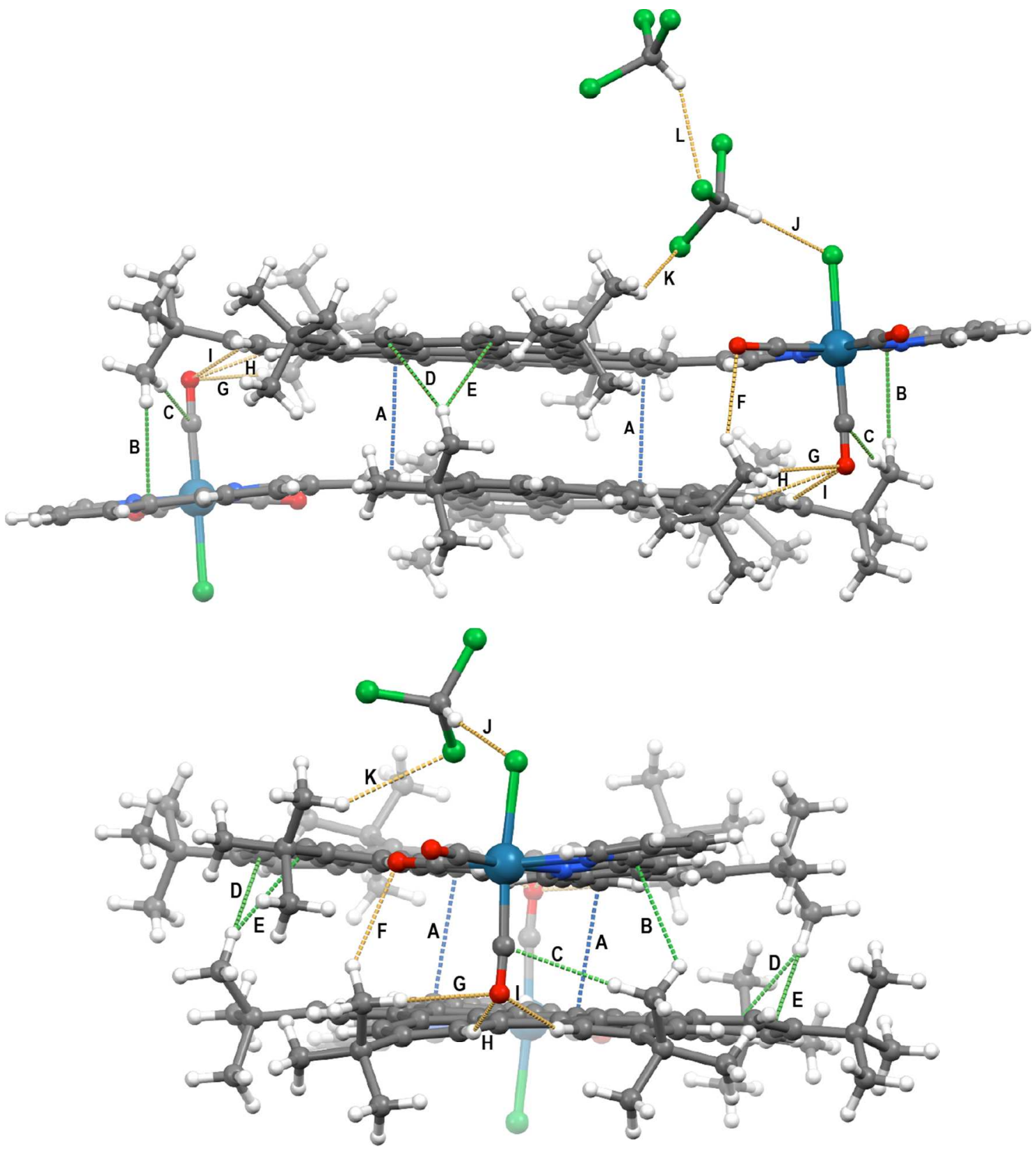

Figure S1. Crystallographic packing diagrams for $\mathbf{4 a}_{3} \mathrm{CHCl}_{3}$ showing selected intermolecular interactions within $\mathrm{HBC}$ dimer pairs and with chloroform. Close contacts $(\AA): \mathrm{C}(\pi) \cdots \mathrm{C}(\pi) 3.308 \mathrm{~A} ; \mathrm{C}\left(s p^{3}\right)-\mathrm{H} \cdots \mathrm{C}(\pi) 2.885 \mathrm{~B}, 2.794 \mathrm{C}, 2.878 \mathrm{D}, 2.379 \mathrm{E} ; \mathrm{C}\left(s p^{3}\right)-\mathrm{H} \cdots \mathrm{O}$ 2.698 F, $2.557 \mathrm{G} ; \mathrm{C}\left(s p^{2}\right)-\mathrm{H} \cdots \mathrm{O} 2.718 \mathrm{H}, 2.685 \mathrm{I} ; \mathrm{C}\left(s p^{3}\right)-\mathrm{H} \cdots \mathrm{Cl} 2.338 \mathrm{~J}, 2.926 \mathrm{~K}, 2.897 \mathrm{~L}$. 


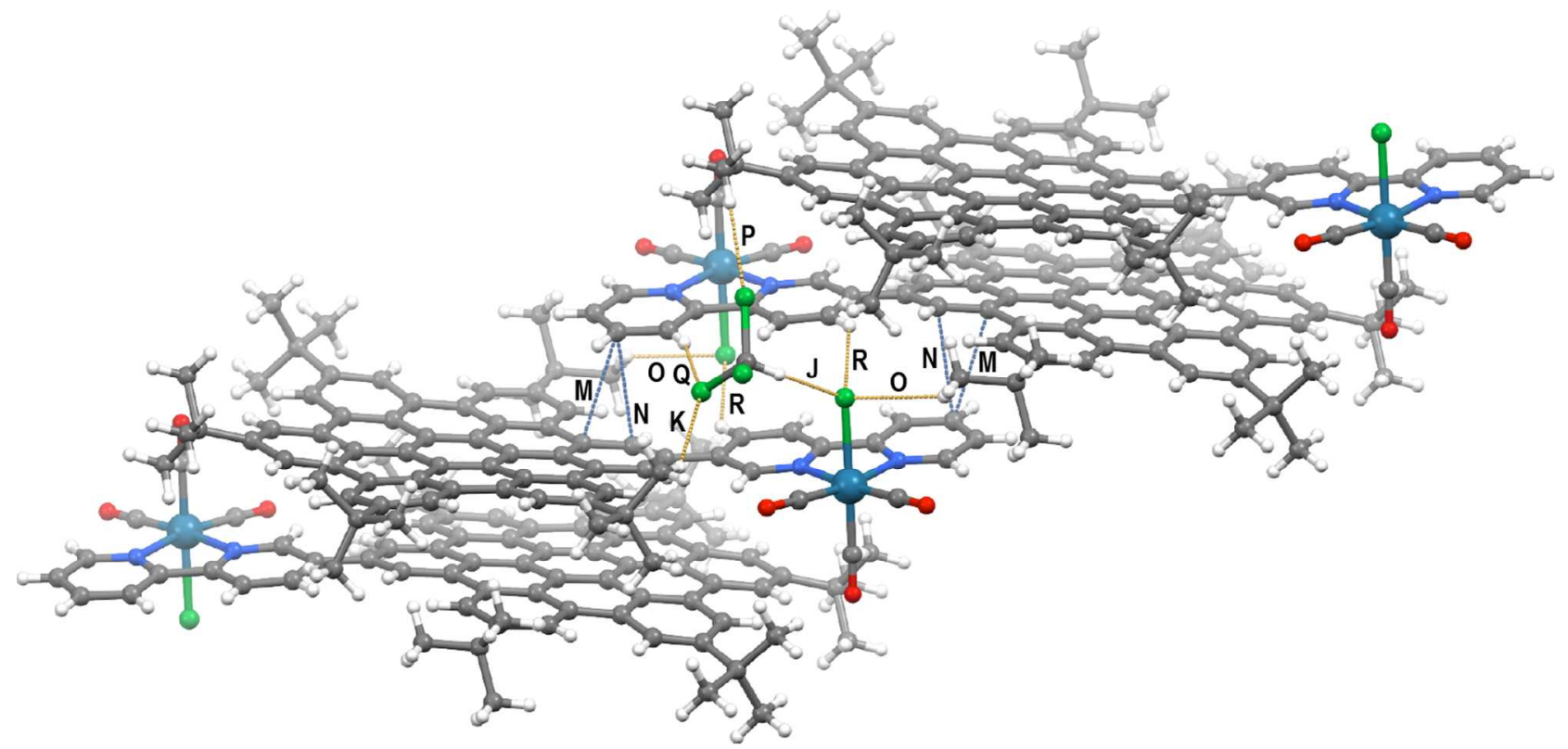

Figure S2. Crystallographic packing diagrams for $4 \mathbf{a} \cdot 3 \mathrm{CHCl}_{3}$ showing selected intermolecular interactions between $\mathrm{HBC}$ dimer pairs and chloroform. Close contacts $(\AA ̊): C(\pi) \cdots C(\pi) 3.353 \mathrm{M}, 3.384 \mathrm{~N} ; \mathrm{C}\left(s p^{3}\right)-\mathrm{H} \cdots \mathrm{Cl} 2.338 \mathrm{~J}, 2.926 \mathrm{~K}, 2.8560,2.698$ P; $\mathrm{C}\left(s p^{2}\right)-\mathrm{H} \cdots \mathrm{Cl} 2.858$ Q, 2.657 R.

\section{Calculations}

Calculations on the structures using the bpy- $\mathrm{HBC}(\mathrm{Me})_{5}$ can be used to provide insight into the structural and electronic properties of these materials. It was found that calculations using a B3LYP functional with 6-31G(d) basis set on all atoms except Re (which required a LANL2DZ ECP) can predict the Raman spectrum of the compounds with a mean absolute deviation in frequencies of $<9 \mathrm{~cm}^{-1}$. 


\section{Spectroscopic Data}
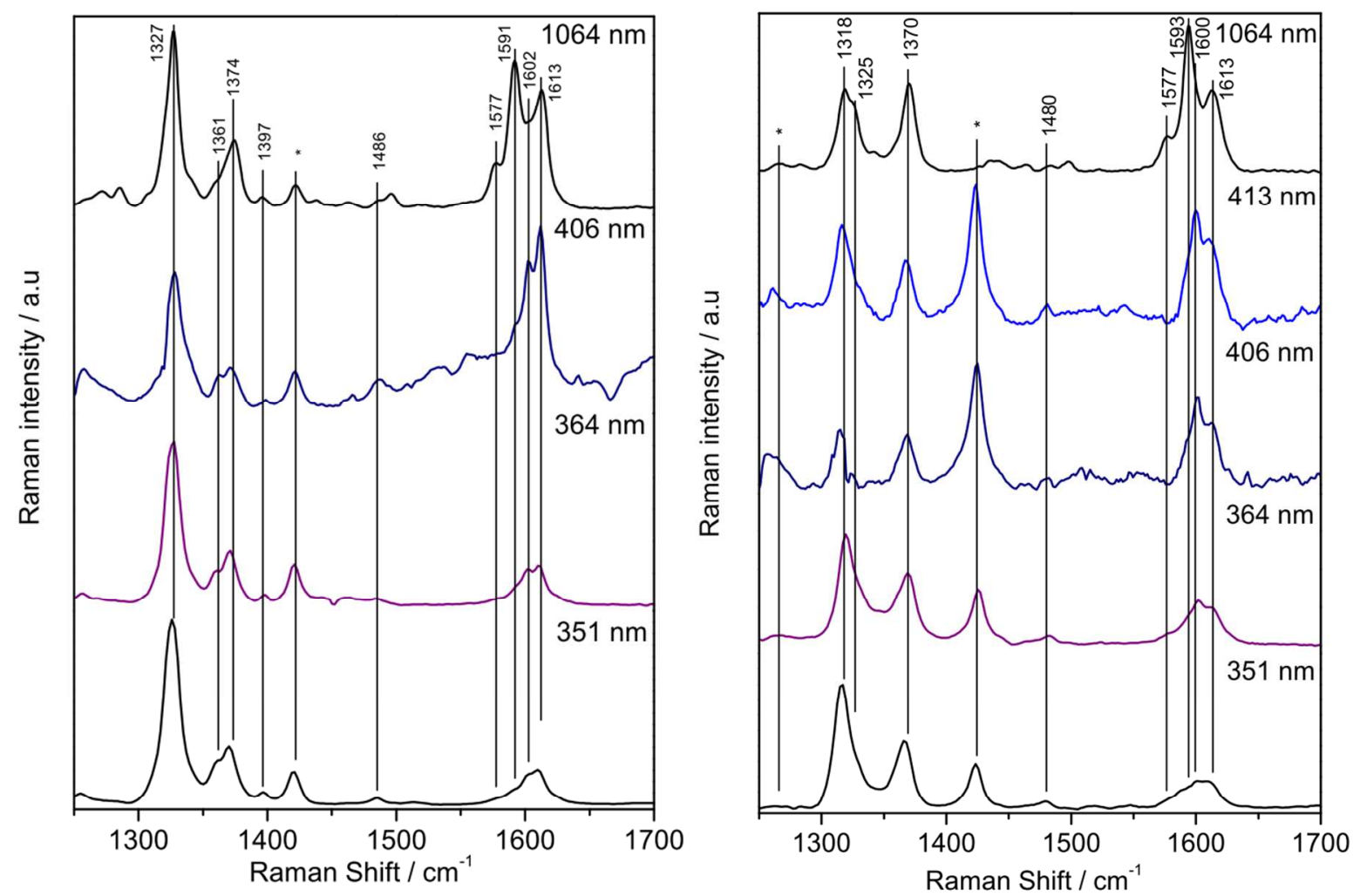

Figure S3. Raman and resonance Raman spectra of the ligands. bpy- $\mathrm{HBC}\left({ }^{\mathrm{t}} \mathrm{Bu}\right)_{5}$ (left box), bpy- $\mathrm{HBC}\left(\mathrm{C}_{12} \mathrm{H}_{25}\right)_{5}($ right box). Resonance Raman spectra acquired in $\mathrm{CH}_{2} \mathrm{Cl}_{2}$ excitation wavelengths shown. Normal Raman spectrum $\left(\lambda_{\text {exc }}=1064 \mathrm{~nm}\right) \mathrm{measured}$ on solid sample. 


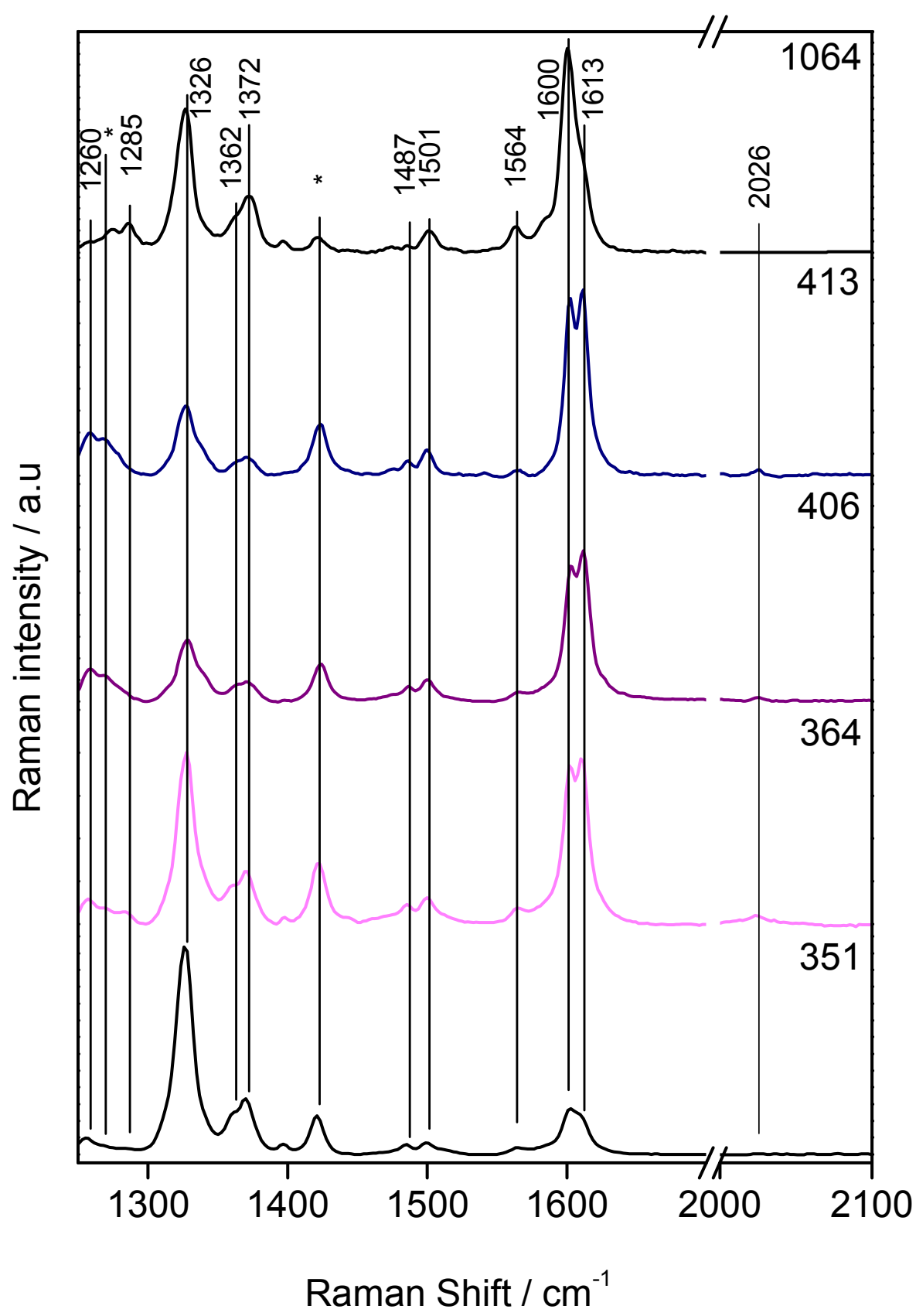

Figure $\mathrm{S}_{4}$. Resonance Raman and normal Raman spectra of the complex $\operatorname{Re}(\mathrm{CO})_{3} \mathrm{Cl}\left(\mathrm{bpy}-\mathrm{HBC}\left(\mathrm{C}_{12} \mathrm{H}_{25}\right)_{5}\right)$. Resonance Raman spectra acquired in $\mathrm{CH}_{2} \mathrm{Cl}_{2}$ excitation wavelengths shown. Normal Raman spectrum $\left(\lambda_{\text {exc }}=1064 \mathrm{~nm}\right)$ measured on solid sample. 

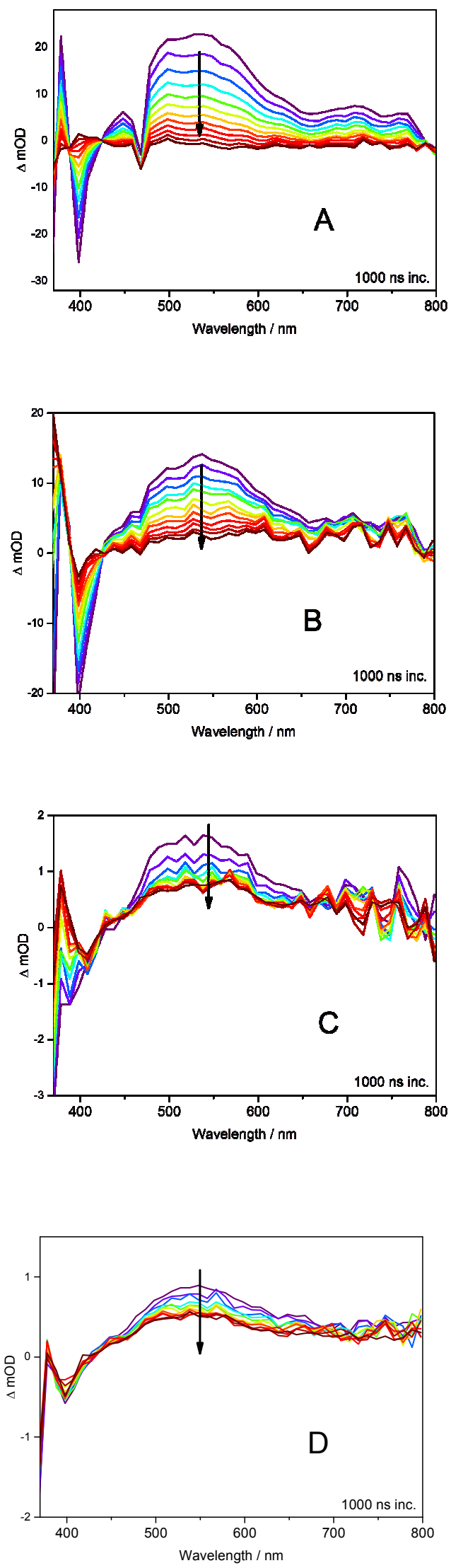

Figure $\mathrm{S}_{\mathbf{5}}$. Transient absorption spectra for samples: A bpy$\mathrm{HBC}\left(\mathrm{C}_{12} \mathrm{H}_{25}\right)_{5}$ $\mathrm{B}$ bpy- $\mathrm{HBC}\left({ }^{\mathrm{t}} \mathrm{Bu}\right)_{5}$;

$\mathrm{C}$ $\mathrm{ReCl}(\mathrm{CO})_{3}\left(\right.$ bpy- $\left.\mathrm{HBC}\left({ }^{\mathrm{t}} \mathrm{Bu}\right)_{5}\right) ; \mathrm{D} \mathrm{ReCl}(\mathrm{CO})_{3}\left(\right.$ bpy- $\left.\mathrm{HBC}\left(\mathrm{C}_{12} \mathrm{H}_{25}\right)_{5}\right)$ in THF $\left(20^{\circ} \mathrm{C}\right)$ concentration $0.02 \mathrm{mM}$. 

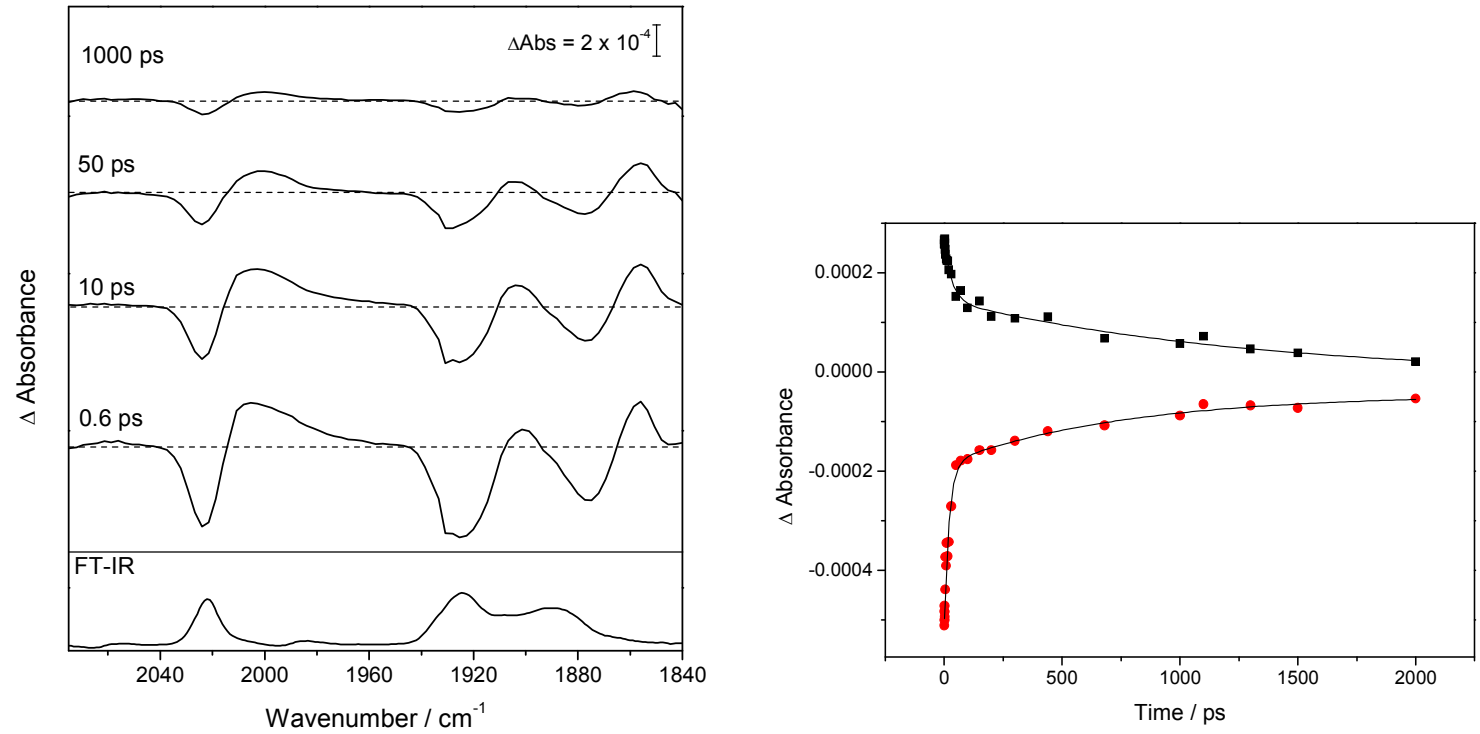

Figure S6. (a) TRIR spectra of $\operatorname{ReCl}(\mathrm{CO})_{3}\left(\mathrm{bpy}-\mathrm{HBC}(\mathrm{tBu})_{5}\right)$ at a number of time delays after excitation at $400 \mathrm{~nm}$ in cyclohexane. (b) Decay of the prominent excited state feature centered at $2000 \mathrm{~cm}^{-1}$ and the parent at $2024 \mathrm{~cm}^{-1}$.
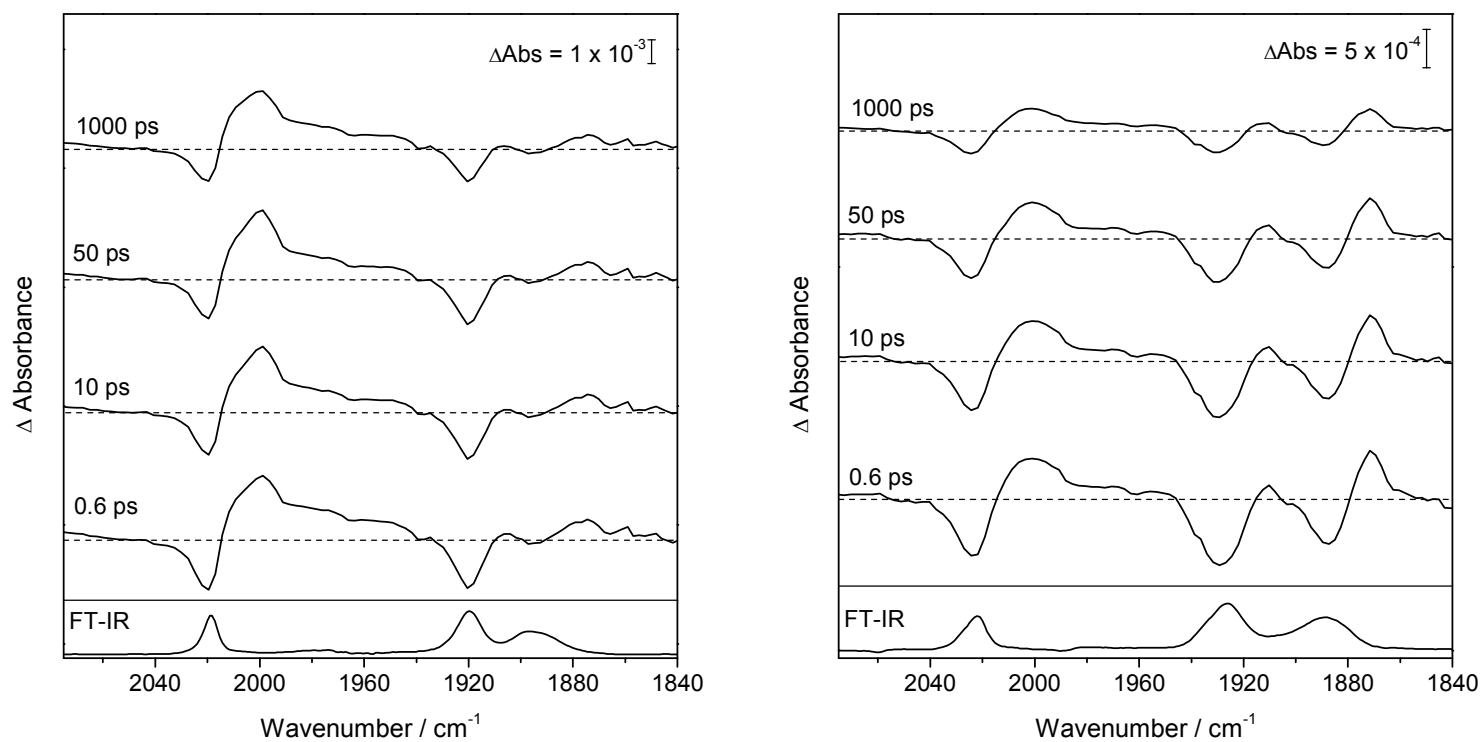

Figure $\mathrm{S}_{7}$. TRIR spectra of $\operatorname{ReCl}(\mathrm{CO})_{3}\left(\mathrm{bpy}-\mathrm{HBC}\left(\mathrm{C}_{12} \mathrm{H}_{22}\right)_{5}\right)$ at a number of time delays after excitation at $400 \mathrm{~nm}$. (a) In THF. (b) In cyclohexane.

\section{References}

(1) Gregg, D. J.; Ollagnier, C. M. A.; Fitchett, C. M.; Draper, S. M. Chem.-Eur. J. 2006, 12, 3043.

(2) Ito, S.; Wehmeier, M.; Brand, J. D.; Kübel, C.; Epsch, R.; Rabe, J. P.; Müllen, K. Chem.-Eur. J. $2000,6,4327$.

(3) Querol, M.; Bozic, B.; Salluce, N.; Belser, P. Polyhedron 2003, 22, 655.

(4) Clar, E. The Aromatic Sextet; John Wiley \& Sons Ltd: London, 1972 